\title{
Asymptotic Bounds on the Rate of Locally Repairable Codes
}

\author{
Ron M. Roth
}

\begin{abstract}
New asymptotic upper bounds are presented on the rate of sequences of locally repairable codes (LRCs) with a prescribed relative minimum distance and locality over a finite field $F$. The bounds apply to LRCs in which the recovery functions are linear; in particular, the bounds apply to linear LRCs over $F$. The new bounds are shown to improve on previously published results, especially when the repair groups are disjoint, namely, they form a partition of the set of coordinates.
\end{abstract}

Index Terms-Large deviations, Linear-programming bound, Locally repairable codes, Sphere-packing bound.

\section{INTRODUCTION}

Hereafter, we fix $F$ to be a finite field $\mathbb{F}_{q}$ (of size $q$ ). For a positive integer $a$, we denote by $[a]$ the integer set $\{1,2, \ldots, a\}$. For a word (vector) $\boldsymbol{y} \in F^{N}$ and a nonempty subset $\mathcal{R} \subseteq[N]$, we let $(\boldsymbol{y})_{\mathcal{R}}$ denote the sub-word of $\boldsymbol{y}$ that is indexed by $\mathcal{R}$.

An $(N, M, d)$ code of length over $F$ is a nonempty subset $\mathrm{C} \subseteq F^{N}$ of size $M$ and minimum (Hamming) distance $d$. The rate of the code is $R=\left(\log _{q} M\right) / N$ and its relative minimum distance (r.m.d.) is $d / N$. When $C$ is linear over $F$ we will use the standard notation $[N, k, d]$ where $k=\log _{q} M$ is the dimension of $\mathrm{C}$. For a nonempty subset $\mathcal{R} \subseteq[N]$, we let $(\mathrm{C})_{\mathcal{R}}$ be the punctured code $\left\{(\boldsymbol{c})_{\mathcal{R}}: c \in C\right\}$.

We say that an $(N, M, d)$ code $\mathrm{C}$ over $F$ is a locally repairable code (in short, LRC) with locality $r$ if for every coordinate $j \in[N]$ there is a subset

$$
\mathcal{R}_{j}=\mathcal{R}_{j}^{*} \bigcup\{j\} \subseteq[N]
$$

of size at most $n=r+1$ (that contains $j$ ) such that for every codeword $c=\left(c_{t}\right)_{t \in[N]}$, the value $c_{j}$ is uniquely determined from $(\boldsymbol{c})_{\mathcal{R}_{j}^{*}}$. In other words, there is a recovery function $\varphi_{j}$ : $F^{\left|\mathcal{R}_{j}^{*}\right|} \rightarrow F$ such that

$$
c_{j}=\varphi_{j}\left((\boldsymbol{c})_{\mathcal{R}_{j}^{*}}\right) .
$$

The set $\mathcal{R}_{j}$ is called the repair group of $j$ and $(\mathrm{C})_{\mathcal{R}_{j}}$ is the respective constituent code. Clearly, the constituent code (C) $\mathcal{R}_{j}$ cannot contain two codewords that differ only on position $j$. Repair groups will usually be represented as a list $\left(\mathcal{R}_{j}\right)_{j=1}^{N}$, where each repair group is indexed by the coordinate $j \in[N]$ that it corresponds to 1

This work was supported by Grants 1396/16 and 1713/20 from the Israel Science Foundation.

Ron M. Roth is with the Computer Science Department, Technion, Haifa 3200003, Israel. Email: ronny @ cs.technion.ac.il

${ }^{1}$ Occasionally, however, we will refer to the set of distinct repair groups within this list; we will then use the notation $\left\{\mathcal{R}_{j}\right\}_{j \in[\ell]}$, where $\ell$ is the number of such repair groups (this notation implicitly assumes that the first $\ell$ coordinates of $\mathrm{C}$ are associated with distinct repair groups). Note that a list of repair groups of an LRC C-and, therefore, the set of distinct repair groups in such a list-may not be unique. Yet such a list (or set) always covers all the coordinates of $\mathrm{C}$.
When the recovery functions $\varphi_{j}$ are linear over $F$ for all $j \in[N]$, we say that the LRC C is linearly recoverable. In this case, each constituent code $(\mathrm{C})_{\mathcal{R}_{j}}$ is a subcode of a linear $\left[\left|\mathcal{R}_{j}\right|,\left|\mathcal{R}_{j}\right|-1\right]$ code over $F$. If $C$ is a linear code over $F$ then it is also linearly recoverable [2, Lemma 10]. The LRC C is said to be all-disjoint if it has a list of repair groups $\left(\mathcal{R}_{j}\right)_{j=1}^{N}$ that satisfies $\mathcal{R}_{j} \cap \mathcal{R}_{j^{\prime}} \in\left\{\emptyset, \mathcal{R}_{j}, \mathcal{R}_{j^{\prime}}\right\}$ for all $j, j^{\prime} \in[N]$. By possibly adding dummy elements to repair groups, this definition is equivalent to requiring that no two distinct repair groups intersect; in this case, the set of distinct repair groups $\left\{\mathcal{R}_{j}\right\}_{j}$ forms a partition of $[N]$. In the all-disjoint case, each repair group $\mathcal{R}_{j}$ is also a repair group for all $j^{\prime} \in \mathcal{R}_{j}$ and, so, each constituent code has minimum distance $\geq 2$.

The general study of LRCs was initiated in [10] and [21], and has generated an extensive body of literature since, including constructions of LRCs, bounds on their parameters, and studies of additional attributes, such as availability and local minimum distance of the constituent codes; see [2], [3], [7], [8], [11], [12], [13], [14], [15], [19], [20], [22], [26], [27], [28].

In this work, we will be interested in asymptotic upper bounds on the rate of linearly recoverable LRCs with prescribed r.m.d. and locality, as the code length tends to infinity 2

Hereafter, by an infinite sequence of codes over $F$ we mean a sequence $\left(\mathrm{C}_{i}\right)_{i=1}^{\infty}$, where each $\mathrm{C}_{i}$ is an $\left(N_{i}, M_{i}, d_{i}\right)$ code over $F$ and the length sequence $\left(N_{i}\right)_{i=1}^{\infty}$ is strictly increasing. The rate and the r.m.d. of the sequence are defined, respectively, as $R=\varlimsup_{\overline{\lim }} \rightarrow \infty R_{i}$ and $\delta=\underline{\lim }_{i \rightarrow \infty} d_{i} / N_{i}$. The supremum over all the rates of sequences of codes with r.m.d. $\geq \delta$ will be denoted by $R_{\text {opt }}(\delta)$. Given $\omega \in[0,1]$, we let $R_{\text {opt }}(\delta, \omega)$ be such a supremum for sequences $\left(C_{i}\right)_{i=1}^{\infty}$ of constant-weight codes, with the codewords of each $\mathrm{C}_{i}$ all having the same weight $w_{i}$, such that $\lim _{i \rightarrow \infty} w_{i} / N_{i}=$ $\omega$. There are several known upper bounds on $R_{\mathrm{opt}}(\delta)$ and $R_{\text {opt }}(\delta, \omega)$, and the best known are obtained using the linearprogramming method [1], [4], [18]. In particular, it is known that $R_{\text {opt }}(\delta, \omega)=R_{\text {opt }}(\delta)=0$ when $\delta \geq(q-1) / q$; hence, we will implicitly assume hereafter that $\delta<(q-1) / q$. It is also easy to see that $R_{\mathrm{opt}}(\delta, \omega)=0$ when $\omega<\delta / 2$. The upper bounds on the rate of LRCs which are considered in this work will often be expressions that involve $R_{\mathrm{opt}}(\delta)$ or $R_{\mathrm{opt}}(\delta, \omega)$; concrete upper bounds for LRCs can then be obtained by replacing these terms with any upper bound on them.

A $(\delta, n)$-LRC sequence over $F$ is an infinite sequence of LRCs $\left(C_{i}\right)_{i=1}^{\infty}$ over $F$ with r.m.d. $\geq \delta$ where each LRC

\footnotetext{
${ }^{2}$ While our results are stated for linearly recoverable LRCs, we shall only use the fact that each recovery function $\varphi_{j}$ in 1 preserves the addition of the field $F$. Hence, our results apply more generally to the case where $F$ is a finite Abelian group and each $\varphi_{j}$ is a homomorphism from $F^{\left|\mathcal{R}_{j}^{*}\right|}$ to $F$.
} 
$\mathrm{C}_{i}$ has locality $r=n-1$. The sequence is said to be alldisjoint if each $C_{i}$ is all-disjoint, and it is linearly recoverable (respectively, linear) if so is each $C_{i}$. It follows from the results of [10] and [21] that the rate $R$ of any $(\delta, n)$-LRC sequence is bounded from above by

$$
R \leq \frac{n-1}{n} \cdot(1-\delta)
$$

This bound, which is oblivious to the field size, can be seen as the LRC counterpart of the Singleton bound, and it applies generally to $(\delta, n)$-LRC sequences (which are not necessarily all-disjoint or linearly recoverable). The bound (2) was improved in [8, Theorem 1] to $R \leq R_{\mathrm{CM}}(\delta, n)$, where

$$
R_{\mathrm{CM}}(\delta, n)=\min _{\tau \in[0,1-\delta]}\left\{\tau \cdot \frac{n-1}{n}+(1-\tau) \cdot R_{\mathrm{opt}}\left(\frac{\delta}{1-\tau}\right)\right\} \text {. }
$$

This bound, which depends on the field size, coincides with 2 when $\delta \mapsto R_{\mathrm{opt}}(\delta)$ is taken to be the Singleton bound $\delta \mapsto 1-\delta$. Small improvements to (3) were obtained in [2]; the latter paper considered the more general setting where the constituent codes $(\mathrm{C})_{\mathcal{R}_{j}}$ have a prescribed minimum distance $\rho \geq 2$ (see also [12]). For non-asymptotic improvements, see [28].

For reference, we also mention the Gilbert-Varshamov-type lower bound on the largest attainable rate of LRC sequences: it was shown in [8, Theorem 2] and [25, Theorem B] that there exists an all-disjoint linear $(\delta, R)$-LRC sequence of rate

$$
R \geq R_{0}(\delta, n)=\frac{n-1}{n}-\lambda(\delta, n),
$$

where $\lambda(\omega, n)$ is defined for every $n \in \mathbb{Z}^{+}$and $\omega \in \mathbb{R}_{\geq 0}$ by

$$
\begin{aligned}
\lambda(\omega, n) & =\lambda_{q}(\omega, n)=\inf _{z \in(0,1]}\left\{-\omega \log _{q} z-\frac{1}{n}\right. \\
+ & \left.\frac{1}{n} \log _{q}\left((1+(q-1) z)^{n}+(q-1)(1-z)^{n}\right)\right\} .
\end{aligned}
$$

The function $\omega \mapsto R_{0}(\omega, n)$ will play a role in our results as well. The expression $\lambda(\omega, n)$ is the growth rate of the volume of a ball of radius $\omega N$ in the subspace of $F^{N}$ formed by the Cartesian product of copies of the $[n, n-1,2]$ parity code over $F$, as $N \rightarrow \infty 3$ The function $\omega \mapsto \lambda_{q}(\omega, n)$ is drawn in Figure 1 for $(q, n)=(2,4),(2,5),(4,5)$; it will follow from our analysis that it is continuous, increasing, and concave on $\left[0^{+}, \infty\right]$ and its values range from 0 at $\omega=0$ to $(n-1) / n$ at $\omega \geq(q-1) / q$. As we show in Lemma 18 in Appendix B finding the infimum in (5) is computationally easy: it amounts to finding a root of a certain real polynomial of degree $\leq n$.

Our first set of results pertain to linearly recoverable LRC sequences that are all-disjoint. We prove the next boundenhancement theorem by using, inter alia, the generalization of [16] to the shortening method for improving upper bounds on the rate of code sequences.

Theorem 1. Let $\delta \mapsto R_{\mathrm{LRC}}(\delta, n)$ be an upper bound on the rate of any all-disjoint linearly recoverable $(\delta, n)-L R C$

\footnotetext{
${ }^{3}$ The proof of the lower bound (4) only requires $\lambda(\omega, n)$ to be an upper bound on this growth rate-a relation which follows from the Chernoff bound as in Eq. 10 below. On the other hand, for our results, we will need a lower bound on this growth rate; to this end, we will use a stronger result from large deviations theory (Eq. 11).
}

sequence over $F$. Then the rate of such a sequence is bounded from above also by

$$
\begin{array}{r}
R_{1}(\delta, n)=\inf _{\tau \in(0,1)} \min _{\left(\theta, \theta^{\prime}\right)}\left\{\tau \cdot R_{0}\left(\frac{\theta}{2}, n\right)\right. \\
\left.+(1-\tau) \cdot R_{\mathrm{LRC}}\left(\theta^{\prime}, n\right)\right\},
\end{array}
$$

where $R_{0}(\cdot, n)$ is defined in (4)-(5) and the (inner) minimum is taken over all pairs $\left(\theta, \theta^{\prime}\right)$ in $[0,(q-1) / q]^{2}$ such that

$$
\tau \cdot \theta+(1-\tau) \cdot \theta^{\prime}=\delta
$$

In particular, Theorem 1 holds for $R_{\mathrm{LRC}}(\delta, n)=R_{\mathrm{opt}}(\delta)$ (i.e., ignoring locality or linear recoverability). This, in turn, yields a concrete upper bound for all-disjoint linearly recoverable LRC sequences. When we do so and substitute $\theta=0$ in the objective function in (6), we get the expression $R_{\mathrm{CM}}(\delta, n)$ in (3). Yet, generally, the minimum in (6) is obtained at some nonzero $\theta$, thereby yielding an improvement. For $\tau \rightarrow 1$ (which forces $\theta=\delta$ ), the objective function in (6) becomes the asymptotic version of the sphere-packing bound of [28] (see Theorem 9 below):

$$
R \leq \boldsymbol{R}_{\mathrm{SP}}(\delta, n)=R_{0}\left(\frac{\delta}{2}, n\right) .
$$

Thus, the objective function in (6) can also be written as:

$$
\tau \cdot R_{\mathrm{SP}}(\theta, n)+(1-\tau) \cdot R_{\mathrm{LRC}}\left(\theta^{\prime}, n\right) .
$$

Remark 1. Theorem 1 can be given the following geometric interpretation (see [16, pp. 77]): for any distinct $\theta_{1}, \theta_{2} \in$ $[0,(q-1) / q]$, the line in the $(\delta, R)$-plane through the points $\left(\theta_{1}, R_{\mathrm{SP}}\left(\theta_{1}\right)\right)$ and $\left(\theta_{2}, R_{\mathrm{LRC}}\left(\theta_{2}, n\right)\right)$ is an upper bound on the rate for any $\delta \in\left[\min \left\{\theta_{1}, \theta_{2}\right\}, \max \left\{\theta_{1}, \theta_{2}\right\}\right]$. In particular, if $\delta \mapsto R_{\mathrm{LRC}}(\delta, n)$ is convex, then, from the convexity of $\delta \mapsto \boldsymbol{R}_{\mathrm{SP}}(\delta, n)$ we get that the lower convex envelope of $\min \left\{R_{\mathrm{SP}}(\delta, n), R_{\mathrm{LRC}}(\delta, n)\right\}$ is also an upper bound.

Our second main result is the following bound.

Theorem 2. The rate of any all-disjoint linearly recoverable $(\delta, n)$-LRC sequence over $F$ is bounded from above by

$$
R_{2}(\delta, n)=\min _{\omega \in[\delta / 2,(q-1) / q]}\left\{R_{0}(\omega, n)+R_{\mathrm{opt}}(\delta, \omega)\right\} .
$$

The bound of Theorem 2 can be further improved by substituting $R_{\mathrm{LRC}}(\delta, n)=R_{2}(\delta, n)$ in Theorem 1

The various bounds are plotted in Figures 2 and 3 for $q=2$ and $n=3,4$ :

- curve (a) presents $\delta \mapsto R_{\mathrm{SP}}(\delta, n)$;

- curve (b) presents $\delta \mapsto R_{\mathrm{CM}}(\delta, n)$, where we have substituted the linear-programming bound $R_{\mathrm{LP}}(\delta)$ of [18] for $\boldsymbol{R}_{\mathrm{opt}}(\delta)$;

- curve (c) presents $\delta \mapsto R_{1}(\delta, n)$ taking $R_{\mathrm{LRC}}(\delta, n)=$ $R_{\mathrm{LP}}(\delta)$ in (6) (the same curve is obtained also for $\left.R_{\mathrm{LRC}}(\delta, n)=R_{\mathrm{CM}}(\delta, n)\right)$;

- curve (d) presents $\delta \mapsto R_{2}(\delta, n)$;

- curve (e) presents $\delta \mapsto R_{1}(\delta, n)$ taking $R_{\mathrm{LRC}}(\delta, n)=$ $R_{2}(\delta, n)$ in (6);

- and curve (f) presents the lower bound $\delta \mapsto R_{0}(\delta, n)$. 


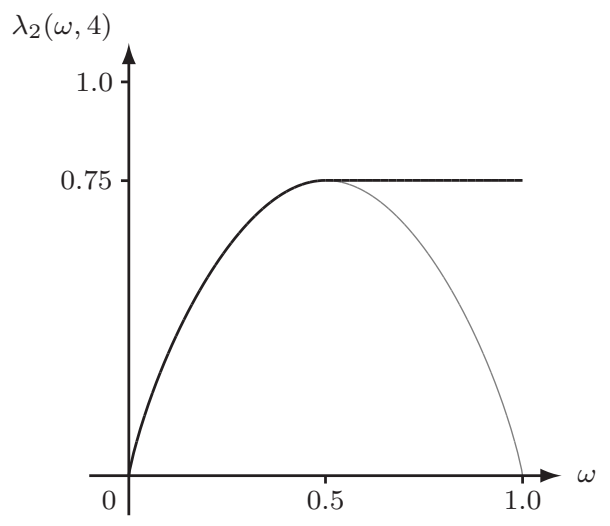

(a)

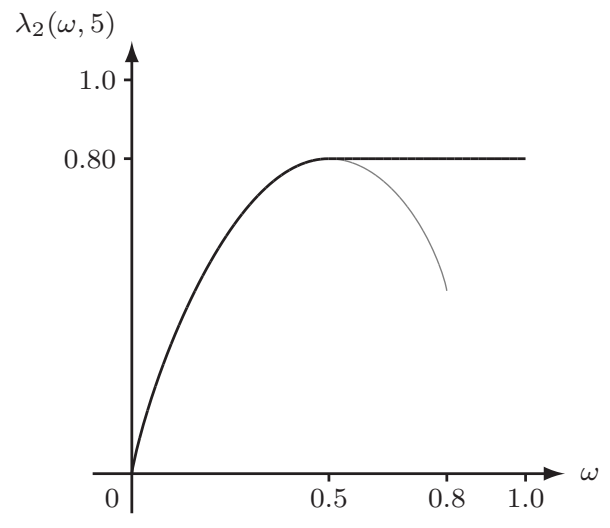

(b)

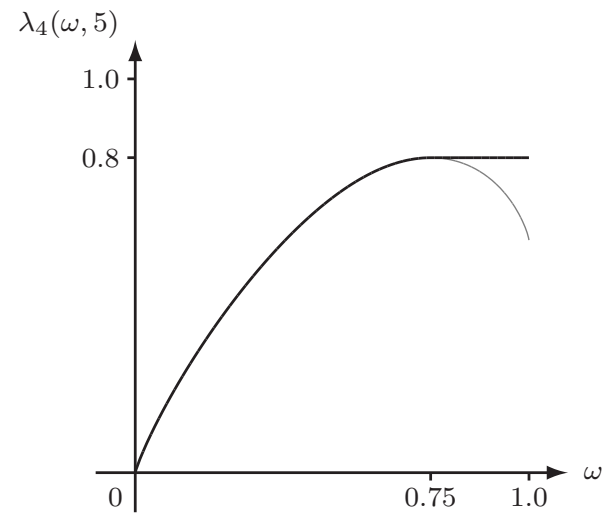

(c)

Fig. 1. Function $\omega \mapsto \lambda_{q}(\omega, n)$ for (a) $(q, n)=(2,4)$, (b) $(q, n)=(2,5)$, and (c) $(q, n)=(4,5)$. The lighter curves depict the function $\omega \mapsto \lambda_{q}^{*}(\omega, n)$.

In the range where any of the curves (b)-(d) is not seen it coincides with curve (e). The values of the upper bounds for $q=2, n=4$, and $\delta \in\{0.07,0.10,0.15,0.30\}$ are summarized in Table [. Notice that curve (d) is not convex and that there is a (small) range where it is worse than curve (b), but curve (e) yields the best results. The bounds of [2] slightly improve on $R_{\mathrm{CM}}(\delta, n)$ but are too close to it to be seen at the scale of the figures.

TABLE I

VALUES OF THE BOUNDS FOR $q=2$ AND $n=4$

\begin{tabular}{cccccc}
\hline \hline$\delta$ & $(\mathrm{a})$ & $(\mathrm{b})$ & $(\mathrm{c})$ & $(\mathrm{d})$ & $(\mathrm{e})$ \\
\hline 0.07 & 0.6133 & 0.6317 & 0.6131 & 0.6079 & 0.6079 \\
0.10 & 0.5681 & 0.5809 & 0.5643 & 0.5576 & 0.5576 \\
0.15 & 0.5004 & 0.4964 & 0.4830 & 0.4781 & 0.4781 \\
0.30 & 0.3346 & 0.2427 & 0.2391 & 0.2470 & 0.2391 \\
\hline \hline
\end{tabular}

Our second set of results includes (weaker) counterparts of Theorems 1 and 2 that apply generally to linearly recoverable LRC sequences (that are not necessarily all-disjoint).

The rest of this work is organized as follows. In Section $\amalg$ we present some basic tools from large deviations theory which are tailored to our needs. Additional tools will be presented in Section III where we also prove the asymptotic sphere-packing bound (8). Section IV is devoted to proving Theorems 1 and 2 . Then, in Section $\nabla$, we present improved results for the special case $q=2$ and $n=3$. Finally, in Section VI we present our bounds for $(\delta, n)$-LRC sequences that are not necessarily all-disjoint (but are still linearly recoverable).

\section{LARGE DEVIATION TOOLS}

We summarize here several basic notions from large deviations theory (see [9, Sections 2.1.2 and 2.2]). Let $X$ be a random variable which takes values in a finite subset $\mathcal{X} \subseteq \mathbb{R}$, with $\operatorname{Prob}\{X=x\}=p(x)>0$ for every $x \in \mathcal{X}$. For every $u \in \mathbb{R}$, let the function $g_{u}:(0,1] \rightarrow \mathbb{R}$ be defined by

$$
g_{u}(z)=z^{-u} \cdot \mathbb{E}\left\{z^{X}\right\}=\sum_{x \in \mathcal{X}} p(x) \cdot z^{x-u}
$$

and let

$$
\gamma(u)=\gamma_{X}(u)=\inf _{z \in(0,1]} g_{u}(z)
$$

The following theorem follows from the Chernoff bound and Cramér's theorem.

Theorem 3. Let $\left(X_{i}\right)_{i=1}^{\infty}$ be a sequence of i.i.d. random variables which take values in a finite subset $\mathcal{X} \subseteq \mathbb{R}$. Then for every real $u \geq x_{\min }=\min _{x \in \mathcal{X}} x$ and $\ell \in \mathbb{Z}^{+}$,

$$
\frac{1}{\ell} \log \operatorname{Prob}\left\{\frac{1}{\ell} \sum_{i=1}^{\ell} X_{i} \leq u\right\} \leq \log \gamma(u) .
$$

Moreover,

$$
\lim _{\ell \rightarrow \infty} \frac{1}{\ell} \log \operatorname{Prob}\left\{\frac{1}{\ell} \sum_{i=1}^{\ell} X_{i} \leq u\right\}=\log \gamma(u) .
$$

Some properties of $u \mapsto \gamma(u)$ are summarized in the next lemma, which is proved in Appendix A

Lemma 4. The function $u \mapsto \gamma(u)$ is

- identically zero when $u<x_{\min }=\min _{x \in \mathcal{X}} x$,

- equal to $p\left(x_{\min }\right)$ when $u=x_{\min }$,

- constant 1 when $u \geq \mathbb{E}\{X\}$,

- strictly increasing when $x_{\min } \leq u<\mathbb{E}\{X\}$,

- continuous when $u \in\left[x_{\min }^{+}, \infty\right)$, and-

- log-concave (i.e., $u \mapsto \log \gamma(u)$ is concave) when $u \geq$ $x_{\min }$.

Let $\mathcal{C}$ be a linear $[n, k]$ code over $F$ and denote by $W_{\mathcal{C}}(z)=$ $\sum_{i=0}^{n} W_{i} z^{i}$ the weight enumerator polynomial of $\mathcal{C}$, namely,

$$
W_{i}=|\{\boldsymbol{c} \in \mathcal{C}: \mathrm{w}(\boldsymbol{c})=i\}|,
$$

where $w(\cdot)$ denotes Hamming weight. For $\omega \in \mathbb{R}$, define

$$
\alpha(\omega)=\alpha_{\mathcal{C}}(\omega)=\inf _{z \in(0,1]}\left\{z^{-n \omega} \cdot W_{\mathcal{C}}(z)\right\} .
$$

We have $\alpha(\omega)=q^{k} \cdot \gamma_{X}(n \omega)$, where $X$ is a random variable which equals the weight of a codeword selected uniformly from $\mathcal{C}$. In particular, $\omega \mapsto \alpha(\omega)$ is continuous on $\mathbb{R}_{\geq 0}$. Moreover, when $\mathcal{C}$ has no trivial coordinates (i.e., none of 
the coordinates is identically zero across all codewords) then $\mathbb{E}\{X\}=n(q-1) / q$; so, $\omega \mapsto \alpha(\omega)$ is strictly increasing (and therefore also positive) at any positive $\omega<(q-1) / q$.

For a code $C$ of length $N$ over $F$ and $\omega \in \mathbb{R}_{\geq 0}$, we denote by $\mathrm{C}(\omega)$ the set of all codewords in $C$ that are contained in a Hamming ball of radius $\omega N$ :

$$
\mathrm{C}(\omega)=\{c \in \mathrm{C}: \mathrm{w}(\boldsymbol{c}) \leq \omega N\} .
$$

Lemma 5. Let $\mathcal{C}$ be a linear code $[n, k]$ over $F$ and for $\ell \in \mathbb{Z}^{+}$, let $\mathbb{C}^{(\ell)}$ be the linear $[\ell n, \ell k]$ code over $F$ defined by the Cartesian product

$$
\mathbb{C}^{(\ell)}=\mathcal{C}^{\times \ell}=\underbrace{\mathcal{C} \times \mathcal{C} \cdots \times \mathcal{C}}_{\ell \text { times }} .
$$

Then the following holds.

(i) For any $\omega \in \mathbb{R}_{\geq 0}$ and $\ell \in \mathbb{Z}^{+}$:

$$
\frac{1}{\ell} \log \left|\mathbb{C}^{(\ell)}(\omega)\right| \leq \log \alpha(\omega)
$$

(ii) For any $\omega \in \mathbb{R}_{\geq 0}$ :

$$
\lim _{\ell \rightarrow \infty} \frac{1}{\ell} \log \left|\mathbb{C}^{(\ell)}(\omega)\right|=\log \alpha(\omega) .
$$

Proof. Apply Theorem 3 with the random variable $X_{i}$ taken as the weight of a codeword selected uniformly from $\mathcal{C}$.

Remark 2. A variant of Lemma 5 holds also for $\mathbb{C}^{(\ell)}(\omega \pm \varepsilon)$, defined as the set of all codewords in $\mathbb{C}^{(\ell)}$ of weight within $(\omega \pm \varepsilon) \ell n$. Assuming that $\mathcal{C}$ has no trivial coordinates, it follows from [9, Theorem 2.1.24] that for any $\omega \in(0,(q-1) / q]$ :

$$
\lim _{\varepsilon \rightarrow 0} \lim _{\ell \rightarrow \infty} \frac{1}{\ell} \log \left|\mathbb{C}^{(\ell)}(\omega \pm \varepsilon)\right|=\log \alpha(\omega) .
$$

For $(q-1) / q<\omega<\max _{\boldsymbol{c} \in \mathcal{C}} \mathrm{w}(\boldsymbol{c}) / n$, this equality holds if we replace $\alpha(\omega)$ by:

$$
\alpha^{*}(\omega)=\inf _{z \in(0,1]} z^{n \omega} \cdot W_{\mathcal{C}}\left(z^{-1}\right)
$$

(this can be shown by stating Theorem 3 with $X_{i}$ and $u$ replaced by $-X_{i}$ and $-u$, respectively).

Example 1. Let $\mathcal{C}$ be the $[n, n-1,2]$ parity code over $F$. The weight enumerator polynomial of the $[n, 1, n]$ repetition code, which is the dual code of $\mathcal{C}$, is given by

$$
W_{\mathcal{C}^{\perp}}(z)=1+(q-1) z^{n}
$$

Hence, by MacWilliams' identities (see [23, Theorem 4.6]):

$$
W_{\mathcal{C}}(z)=\frac{1}{q}\left((1+(q-1) z)^{n}+(q-1)(1-z)^{n}\right) .
$$

Thus, for the code $\mathcal{C}$ we have:

$$
\begin{aligned}
\frac{1}{n} \cdot \log _{q} \alpha(\omega) & =\frac{1}{n} \inf _{z \in(0,1]} \log _{q}\left(z^{-n \omega} \cdot W_{\mathcal{C}}(z)\right) \\
& =\lambda(\omega, n),
\end{aligned}
$$

where $\lambda(\omega, n)$ is defined in (5). From this we can conclude that the mapping $\omega \mapsto \lambda(\omega, n)$ takes the value 0 at $\omega=0$ and $(n-1) / n$ at $\omega=(q-1) / q$, and is strictly increasing in between for any $n>1$. For $\omega \geq(q-1) / q$ it remains a constant $(n-1) / n$.
In Figure 1, we have also depicted the following function, which is defined for $(q-1) / q<\omega<\max _{\boldsymbol{c} \in \mathcal{C}} \mathrm{w}(\boldsymbol{c}) / n$ :

$$
\begin{aligned}
\lambda^{*}(\omega, n)=\lambda_{q}^{*}(\omega, n) & =\frac{1}{n} \cdot \log _{q} \alpha^{*}(\omega) \\
& =\frac{1}{n} \inf _{z \in(0,1]} \log _{q}\left(z^{n \omega} \cdot W_{\mathcal{C}}\left(z^{-1}\right)\right) .
\end{aligned}
$$

Note that $\max _{\boldsymbol{c} \in \mathcal{C}} \mathrm{W}(\boldsymbol{c}) / n$ equals 1 , except when $q=2$ and $n$ is odd, in which case it equals $(n-1) / n$. It is also fairly easy to see that when $q=2$ and $n$ is even we have $\lambda_{2}^{*}(\omega, n)=$ $\lambda_{2}(1-\omega, n)$.

\section{ASYMPTOTIC SPHERE-PACKING BOUND}

The purpose of this section is to present additional tools that will be used in this work. Along the way, we present an asymptotic formulation of the sphere-packing bound of [28] for the all-disjoint linearly recoverable case. The following proposition will be useful for this purpose, as well as for other proofs in the sequel.

Proposition 6. Given $n \in \mathbb{Z}^{+}$, let $\left(\mathrm{A}_{i}\right)_{i=1}^{\infty}$ be an infinite sequence of codes over $F$ where each $\mathrm{A}_{i}$ is a linear code of length $N_{i}$ over $F$ of the form

$$
\mathrm{A}_{i}=\mathcal{C}_{i, 1} \times \mathcal{C}_{i, 2} \times \cdots \times \mathcal{C}_{i, \ell_{i}},
$$

with each constituent code $\mathcal{C}_{i, j}$ being a linear $\left[n_{i, j}, n_{i, j}-1\right]$ code of length $n_{i, j} \leq n$ over $F$. Then for any nonnegative real sequence $\left(\omega_{i}\right)_{i=1}^{\infty}$ such that $\underline{\lim }_{i \rightarrow \infty} \omega_{i}=\omega$ :

$$
\varlimsup_{i \rightarrow \infty} \frac{1}{N_{i}} \log _{q} \frac{\left|\mathrm{A}_{i}\right|}{\left|\mathrm{A}_{i}\left(\omega_{i}\right)\right|} \leq R_{0}(\omega, n),
$$

where $R_{0}(\omega, n)$ is defined in (4)-(5).

\section{A. Proof of Proposition 6}

We will prove Proposition 6 in two steps. We first prove it under an additional assumption on the sequence $\left(\mathrm{A}_{i}\right)_{i=1}^{\infty}$ (Lemma 7). We then prove a more general claim (Lemma 8), which will also be needed in Section VI where we remove the all-disjoint assumption.

Lemma 7. Proposition 6 holds when all the constituent codes have the same length $n_{i, j}=n$.

Proof. The claim is immediate when $\omega=0$, so we assume hereafter in the proof that $\omega>0$.

For $s \in[n]$, let $\mathcal{C}_{s}$ be the linear $[n, n-1]$ code over $F$ with the parity-check matrix

$$
(\underbrace{11 \ldots 1}_{s \text { times }} 00 \ldots 0)
$$

Without real loss of generality we can assume that $\mathcal{C}_{i, j} \in$ $\left\{\mathcal{C}_{s}\right\}_{s=1}^{n}$ for each $j \in\left[\ell_{i}\right]$. Therefore, we can write

$$
\mathrm{A}_{i}=\mathbb{C}_{1}^{\left(\ell_{i, 1}\right)} \times \mathbb{C}_{2}^{\left(\ell_{i, 2}\right)} \times \cdots \times \mathbb{C}_{n}^{\left(\ell_{i, n}\right)}
$$

where $\mathbb{C}_{s}^{(\ell)}=\left(\mathcal{C}_{s}\right)^{\times \ell}$ and $\ell_{i, 1}, \ell_{i, 2}, \ldots, \ell_{i, n}$ are nonnegative integers that sum to $\ell_{i}$. (The code $\mathbb{C}_{s}^{(0)}$ is taken to have zero length, which will practically mean that we remove $\mathbb{C}_{s}^{\left(\ell_{i, s}\right)}$ from the product (13) in case $\ell_{i, s}=0$.) 
For each $s \in[n]$, the weight enumerator polynomial of the dual code of $\mathcal{C}_{s}$ is given by

$$
W_{\mathcal{C}_{s}^{\perp}}(z)=1+(q-1) z^{s}
$$

and, so, by MacWilliams' identities:

$W_{\mathcal{C}_{s}}(z)=\frac{1}{q}(1+(q-1) z)^{n-s}\left((1+(q-1) z)^{s}+(q-1)(1-z)^{s}\right)$.

In particular, for every $z \in(0,1]$ :

$$
W_{\mathcal{C}_{s}}(z) \geq W_{\mathcal{C}_{n}}(z)=\frac{1}{q}\left((1+(q-1) z)^{n}+(q-1)(1-z)^{n}\right) .
$$

Hence, for every $i \in \mathbb{Z}^{+}$:

$$
\log _{q} \alpha_{\mathcal{C}_{s}}\left(\omega_{i}\right) \geq \log _{q} \alpha_{\mathcal{C}_{n}}\left(\omega_{i}\right)=n \cdot \lambda\left(\omega_{i}, n\right) .
$$

Fixing some $\varepsilon \in(0, \omega)$, by Lemma 5 (ii) we then get that, whenever $i$ and $\ell_{i, s}$ are sufficiently large,

$$
\begin{aligned}
\frac{1}{\ell_{i, s} n} \log _{q}\left|\mathbb{C}_{s}^{\left(\ell_{i, s}\right)}\left(\omega_{i}\right)\right| & \geq \frac{1}{\ell_{i, s} n} \log _{q}\left|\mathbb{C}_{s}^{\left(\ell_{i, s}\right)}(\omega-\varepsilon)\right| \\
& \geq \lambda(\omega-\varepsilon, n)-\varepsilon
\end{aligned}
$$

For $i \in \mathbb{Z}^{+}$define

$$
\mathcal{S}(i)=\left\{s \in[n]: \ell_{i, s} \geq \sqrt{\ell_{i}}\right\} .
$$

For sufficiently large $i$ (and, therefore, sufficiently large $\ell_{i}$ ) we have:

$$
\begin{aligned}
& \frac{1}{\ell_{i} n} \log _{q}\left|\mathrm{~A}_{i}\left(\omega_{i}\right)\right| \\
& \geq \frac{1}{\ell_{i} n} \sum_{s=1}^{n} \log _{q}\left|\mathbb{C}^{\left(\ell_{i, s}\right)}\left(\omega_{i}\right)\right| \\
& \geq \sum_{s \in \mathcal{S}(i)} \frac{\ell_{i, s}}{\ell_{i}} \cdot \frac{1}{\ell_{i, s} n} \log _{q}\left|\mathbb{C}^{\left(\ell_{i, s}\right)}\left(\omega_{i}\right)\right| \\
& \geq(\lambda(\omega-\varepsilon, n)-\varepsilon) \sum_{s \in \mathcal{S}(i)} \frac{\ell_{i, s}}{\ell_{i}} .
\end{aligned}
$$

Noting that

$$
1-\frac{n}{\sqrt{\ell_{i}}}<\sum_{s \in \mathcal{S}(i)} \frac{\ell_{i, s}}{\ell_{i}} \leq 1
$$

we conclude that

$$
\varliminf_{i \rightarrow \infty} \frac{1}{\ell_{i} n} \log _{q}\left|\mathrm{~A}_{i}\left(\omega_{i}\right)\right| \geq \lambda(\omega-\varepsilon, n)-\varepsilon .
$$

Finally, we get (12) by taking the limit $\varepsilon \rightarrow 0$ and recalling that $N_{i}=\ell_{i} n$ and that

$$
\frac{1}{\ell_{i} n} \log _{q}\left|\mathrm{~A}_{i}\right|=\frac{n-1}{n} .
$$

Remark 3. It follows from the proof that equality is attained in (12) when $A_{i}=\left(\mathcal{C}_{n}\right)^{\times \ell_{i}}$, namely, the growth rate of $A_{i}(\omega)$ is minimized when each $\mathcal{C}_{i, j}$ is the $[n, n-1,2]$ parity code. This result, however, holds only asymptotically (namely, when $i \rightarrow \infty)$ and not necessarily for individual values of $i$. For example, for $q=2, n=5$, and $\ell_{i}=\ell_{1}=1$ we have $\left|\mathcal{C}_{5}(0.4)\right|=11$ yet $\left|\mathcal{C}_{2}(0.4)\right|=8$ and $\left|\mathcal{C}_{3}(0.4)\right|=7$.
The next lemma involves the following definition. For $n \in$ $\mathbb{Z}^{+}, \omega \in \mathbb{R}_{\geq 0}$, and a real $\mu \in[1, n]$, define:

$$
\bar{R}_{0}(\omega, n, \mu)=\max _{\boldsymbol{\pi}} \min _{\vartheta} \sum_{s \in[n]} \pi_{s} \cdot R_{0}\left(\vartheta_{s}, s\right),
$$

where $R_{0}(\cdot, \cdot)$ is as in (4), the maximum is taken over all vectors $\boldsymbol{\pi}=\left(\pi_{s}\right)_{s \in[n]} \in \mathbb{R}_{\geq 0}^{n}$ that satisfy
P1) $\sum_{s \in[n]} \pi_{s}=1$ and
P2) $\sum_{s \in[n]} \frac{\pi_{s}}{s} \geq \frac{1}{\mu}$,

and the minimum is taken over all vectors $\boldsymbol{\vartheta}=\left(\vartheta_{s}\right)_{s \in[n]} \in$ $\mathbb{R}_{\geq 0}^{n}$ that satisfy

P3) $\sum_{s \in[n]} \pi_{s} \cdot \vartheta_{s}=\omega$.

Remark 4. The expression in (15) is fairly easy to compute, and we provide a formula for it in Proposition 21 in Appendix B In particular, we show there that the support of the maximizing vector $\pi$ in 15 is $\{\lfloor\mu\rfloor,\lceil\mu\rceil\}$; thus, when $\mu$ is an integer then

$$
\bar{R}_{0}(\omega, n, \mu)=R_{0}(\omega, \mu) .
$$

It will also follow from our analysis that for given $\omega \in \mathbb{R}_{\geq 0}$ and $\mu \in[1, \infty)$, the value $\bar{R}_{0}(\omega, n, \mu)$ is the same for all $n \geq \mu$.

Proposition 6 follows by substituting $\mu=n$ in the next lemma.

Lemma 8. Let $\left(\mathrm{A}_{i}\right)_{i=1}^{\infty}$ be as in Proposition 6 and assume in addition that for a prescribed $\mu \in[1, n]$, the average length of the constituent codes satisfies

$$
\varlimsup_{i \rightarrow \infty} \frac{N_{i}}{\ell_{i}} \leq \mu .
$$

Then for any nonnegative real sequence $\left(\omega_{i}\right)_{i=1}^{\infty}$ such that $\underline{\lim }_{i \rightarrow \infty} \omega_{i}=\omega$ :

$$
\varlimsup_{i \rightarrow \infty} \frac{1}{N_{i}} \log _{q} \frac{\left|\mathrm{A}_{i}\right|}{\left|\mathrm{A}_{i}\left(\omega_{i}\right)\right|} \leq \bar{R}_{0}(\omega, n, \mu),
$$

where $\bar{R}_{0}(\omega, n, \mu)$ is defined in (15).

Proof. By possibly permuting the coordinates of $\mathrm{A}_{i}$, we can write

$$
\mathrm{A}_{i}=\mathrm{A}_{i, 1} \times \mathrm{A}_{i, 2} \times \cdots \times \mathrm{A}_{i, n},
$$

where each $\mathrm{A}_{i, s}$ is a linear $\left[s \cdot \ell_{i, s},(s-1) \cdot \ell_{i, s}\right]$ code over $F$ of the form

$$
\mathrm{A}_{i, s}=\mathcal{C}_{i, 1}^{\prime} \times \mathcal{C}_{i, 2}^{\prime} \times \cdots \times \mathcal{C}_{i, \ell_{i, s}}^{\prime},
$$

with each $\mathcal{C}_{i, j}^{\prime}$ being a linear $[s, s-1]$ code over $F$.

Define $\boldsymbol{\pi}_{i}=\left(\pi_{i, s}\right)_{s \in[n]}$ by

$$
\pi_{i, s}=\frac{s \cdot \ell_{i, s}}{N_{i}}
$$

(i.e., $\pi_{i, s}$ is the fraction of coordinates of $\mathrm{A}_{i}$ that correspond to constituent codes of length $s$ ). We have

$$
\sum_{s \in[n]} \pi_{i, s}=\frac{1}{N_{i}} \sum_{s \in[n]} s \cdot \ell_{i, s}=\frac{1}{N_{i}} \sum_{j \in\left[\ell_{i}\right]} n_{i, j}=1
$$


and

$$
\varliminf_{i \rightarrow \infty} \sum_{s \in[n]} \frac{\pi_{i, s}}{s}=\varliminf_{i \rightarrow \infty} \frac{1}{N_{i}} \sum_{s \in[n]} \ell_{i, s}=\varliminf_{i \rightarrow \infty} \frac{\ell_{i}}{N_{i}} \stackrel{16}{\geq} \frac{1}{\mu} .
$$

By possibly restricting to a subsequence of $\left(\mathrm{A}_{i}\right)_{i=1}^{\infty}$, we can assume that the sequence $\left(\boldsymbol{\pi}_{i}\right)_{i=1}^{\infty}$ converges to a limit $\boldsymbol{\pi}=\left(\pi_{s}\right)_{s \in[n]}$; by (18)- -19$]$, this limit satisfies conditions (P1) and $(\mathrm{P} 2)$.

Let $\left(\omega_{i}\right)_{i=1}^{\infty}$ be a nonnegative real sequence such that $\underline{\lim }_{i \rightarrow \infty} \omega_{i}=\omega$ and let $\boldsymbol{\vartheta}=\left(\vartheta_{s}\right)_{s \in[n]}$ be a vector in $\mathbb{R}_{\geq 0}^{n}$ that satisfies condition (P3). For any $i \in \mathbb{Z}^{+}$and $s \in[n]$, define:

$$
\vartheta_{i, s}=\left\{\begin{array}{cl}
\frac{\pi_{s}}{\pi_{i, s}} \cdot \frac{\omega_{i}}{\omega} \cdot \vartheta_{s} & \text { if } \omega>0 \text { and } s \in \operatorname{Supp}\left(\boldsymbol{\pi}_{i}\right) \\
0 & \text { otherwise }
\end{array}\right.
$$

where $\operatorname{Supp}(\cdot)$ denotes the support of a vector. It is easily seen that

$$
\sum_{s \in[n]} \pi_{i, s} \cdot \vartheta_{i, s} \leq \omega_{i}
$$

and that for any $s \in \operatorname{Supp}(\boldsymbol{\pi})$ :

$$
\lim _{i \rightarrow \infty} \vartheta_{i, s}=\vartheta_{s}
$$

We then have:

$$
\frac{1}{N_{i}} \log _{q}\left|\mathrm{~A}_{i}\right| \stackrel{17}{=} \sum_{s \in \operatorname{Supp}\left(\boldsymbol{\pi}_{i}\right)} \pi_{i, s} \cdot \frac{1}{s \cdot \ell_{i, s}} \log _{q}\left|\mathrm{~A}_{i, s}\right|
$$

and

$\frac{1}{N_{i}} \log _{q}\left|\mathrm{~A}_{i}\left(\omega_{i}\right)\right| \stackrel{177+20}{\geq} \sum_{s \in \operatorname{Supp}\left(\boldsymbol{\pi}_{i}\right)} \pi_{i, s} \cdot \frac{1}{s \cdot \ell_{i, s}} \log _{q}\left|\mathrm{~A}_{i, s}\left(\vartheta_{i, s}\right)\right|$.

Hence,

$$
\begin{aligned}
& \varlimsup_{i \rightarrow \infty} \frac{1}{N_{i}} \log _{q} \frac{\left|\mathrm{A}_{i}\right|}{\left|\mathrm{A}_{i}\left(\omega_{i}\right)\right|} \\
& \leq \varlimsup_{i \rightarrow \infty} \sum_{s \in \operatorname{Supp}\left(\pi_{i}\right)} \pi_{i, s} \cdot \frac{1}{s \cdot \ell_{i, s}} \log _{q} \frac{\left|\mathrm{A}_{i, s}\right|}{\left|\mathrm{A}_{i, s}\left(\vartheta_{i, s}\right)\right|} \\
& \quad=\quad \sum_{s \in \operatorname{Supp}(\pi)} \varlimsup_{i \rightarrow \infty} \pi_{i, s} \cdot \frac{1}{s \cdot \ell_{i, s}} \log _{q} \frac{\left|\mathrm{A}_{i, s}\right|}{\left|\mathrm{A}_{i, s}\left(\vartheta_{i, s}\right)\right|} \\
& \quad \leq \sum_{s \in[n]} \pi_{s} \cdot R_{0}\left(\vartheta_{s}, s\right) .
\end{aligned}
$$

Finally, selecting $\vartheta \in \mathbb{R}_{>0}^{n}$ that minimizes the inner expression in 15) (subject to condition (P3)), we get:

$$
\begin{aligned}
\varlimsup_{i \rightarrow \infty} \frac{1}{N_{i}} \log _{q} \frac{\left|\mathrm{A}_{i}\right|}{\left|\mathrm{A}_{i}\left(\omega_{i}\right)\right|} & \leq \min _{\vartheta} \sum_{s \in[n]} \pi_{s} \cdot R_{0}\left(\vartheta_{s}, s\right) \\
& \leq \bar{R}_{0}(\omega, n, \mu),
\end{aligned}
$$

for any $\pi \in \mathbb{R}_{\geq 0}^{n}$ that satisfies conditions (P1)-(P2).

\section{B. Ambient spaces of LRCs}

Let $C$ be a linearly recoverable LRC (which is not necessarily all-disjoint) of length $N$ and locality $r=n-1$ over $F$, and let $\left\{\mathcal{R}_{j}\right\}_{j}$ be a set of distinct repair groups of C. Recall that for each repair group $\mathcal{R}_{j}$, the constituent code $(\mathrm{C})_{\mathcal{R}_{j}}$ is a subcode of a linear $\left[\left|\mathcal{R}_{j}\right|,\left|\mathcal{R}_{j}\right|-1\right]$ code, $\mathcal{C}_{j}$, over $F$. Let $A$ be a largest subset of $F^{N}$ which satisfies the following linear constraints:

$$
(\mathrm{A})_{\mathcal{R}_{j}} \subseteq \mathcal{C}_{j}, \quad j \in[\ell] .
$$

Clearly, A is a linear subspace of $F^{N}$ (which is defined uniquely by (21) and $C \subseteq A$. We will refer hereafter to $A$ as an ambient space of $\mathrm{C}$ (this term is similar to the notion of an $\mathcal{L}$-space defined in [28]).

In the all-disjoint case, we can assume that $\left\{\mathcal{R}_{j}\right\}_{j}$ forms a partition of $[N]$. By possibly permuting the coordinates of both $C$ and $A$, we can further assume that $A$ takes the form

$$
\mathrm{A}=\mathcal{C}_{1} \times \mathcal{C}_{2} \times \cdots \times \mathcal{C}_{\ell}
$$

\section{Sphere-packing bound}

Ambient spaces were used in [28] as an ingredient in obtaining a sphere-packing bound on LRCs. The asymptotic formulation of this bound is presented in the next theorem.

Theorem 9. The rate of any all-disjoint linearly recoverable $(\delta, n)$-LRC sequence over $F$ is bounded from above by

$$
R_{\mathrm{SP}}(\delta, n)=R_{0}\left(\frac{\delta}{2}, n\right)
$$

Proof. Let $\left(\mathrm{C}_{i}\right)_{i=1}^{\infty}$ be an all-disjoint linearly recoverable $(\delta, n)$-LRC sequence, with $N_{i}$ and $d_{i}$ being the length and the minimum distance of $C_{i}$. Letting $A_{i}$ be an ambient space of $C_{i}$, by a sphere-packing argument we get:

$$
\left|\mathrm{C}_{i}\right| \leq \frac{\left|\mathrm{A}_{i}\right|}{\mathrm{A}_{i}\left(d_{i}-1 /\left(2 N_{i}\right)\right)}
$$

The result follows from Proposition 6 by taking $\omega_{i}=$ $\left(d_{i}-1\right) /\left(2 N_{i}\right)$.

Remark 5. For general $\mu \in[1, n]$, we get from Lemma 8 that

$$
\bar{R}_{0}\left(\frac{\delta}{2}, n, \mu\right)
$$

is an upper bound on the rate of any all-disjoint linearly recoverable $(\delta, n)$-LRC sequence $\left(C_{i}\right)_{i=1}^{\infty}$ over $F$, with $\mu$ bounding from above the supremum (as $i \rightarrow \infty$ ) over the average sizes, $N_{i} / \ell_{i}$, of the distinct (and disjoint) repair groups of $C_{i}$. A similar bound can be stated when the average size is computed per coordinate, so that each repair group is counted a number of times equaling its size. See Remark 11 in Appendix B 


\section{Proofs of Theorems 1 and 2}

In this section, we prove Theorems 1 and 2 .

We start with the next simple lemma, usually attributed to Bassalygo or Elias; it provides an effective tool in obtaining upper bounds.

Lemma 10. Let $\mathrm{A}$ be a subspace of $F^{N}$ and let $\mathrm{B}$ and $\mathrm{C}$ be subsets of A. Then there exists $\boldsymbol{y} \in \mathrm{A}$ for which

$$
|(\boldsymbol{y}+\mathrm{C}) \cap \mathrm{B}| \geq \frac{|\mathrm{B}|}{|\mathrm{A}|} \cdot|\mathrm{C}| .
$$

Proof. Let $\chi_{\mathrm{B}}: \mathrm{A} \rightarrow\{0,1\}$ be the characteristic function of $\mathrm{B}$, namely, $\chi(\boldsymbol{y})=1$ when $\boldsymbol{y} \in \mathrm{B}$ and $\chi(\boldsymbol{y})=0$ otherwise. Then,

$$
\begin{aligned}
\frac{1}{|\mathrm{~A}|} \cdot|\mathrm{C}| \cdot|\mathrm{B}| & =\frac{1}{|\mathrm{~A}|} \sum_{\boldsymbol{c} \in \mathrm{C}} \sum_{\boldsymbol{y} \in \mathrm{A}} \chi_{\mathrm{B}}(\boldsymbol{c}+\boldsymbol{y}) \\
& =\frac{1}{|\mathrm{~A}|} \sum_{\boldsymbol{y} \in \mathrm{A}} \sum_{\boldsymbol{c} \in \mathrm{C}} \chi_{\mathrm{B}}(\boldsymbol{y}+\boldsymbol{c}) \\
& =\frac{1}{|\mathrm{~A}|} \sum_{\boldsymbol{y} \in \mathrm{A}}|(\boldsymbol{y}+\mathrm{C}) \cap \mathrm{B}| .
\end{aligned}
$$

Hence,

$$
\max _{\boldsymbol{y} \in \mathrm{A}}|(\boldsymbol{y}+\mathrm{C}) \cap \mathrm{B}| \geq \frac{|\mathrm{B}|}{|\mathrm{A}|} \cdot|\mathrm{C}| .
$$

Proof of Theorem 1$]$ We tailor the generalization of the shortening method of [16, Theorems 1 and 2] to our setting. Let $\left(\mathrm{C}_{i}\right)_{i=1}^{\infty}$ be an all-disjoint linearly recoverable $(\delta, n)$-LRC sequence over $F$ and fix $\tau$ in the interval $(0,1)$ and a pair $\left(\theta, \theta^{\prime}\right) \in[0,(q-1) / q]^{2}$ that satisfies (7). For each $i \in \mathbb{Z}^{+}$, let $N_{i}$ and $d_{i}$ be the length and the minimum distance of $\mathrm{C}_{i}$, respectively, where

$$
\varliminf_{i \rightarrow \infty} \frac{d_{i}}{N_{i}} \geq \delta
$$

Let $\left\{\mathcal{R}_{i, j}\right\}_{j \in\left[\ell_{i}\right]}$ be a set of distinct (and disjoint) repair groups of $\mathrm{C}_{i}$, and denote

$$
t_{i}=\left|\bigcup_{j \in\left[\ell_{i}^{\prime}\right]} \mathcal{R}_{i, j}\right|,
$$

where $\ell_{i}^{\prime}$ is the smallest in $\left[\ell_{i}\right]$ so that $t_{i} \geq \tau N_{i}$; in particular,

$$
\lim _{i \rightarrow \infty} \frac{t_{i}}{N_{i}}=\tau \text {. }
$$

By possibly permuting the coordinates of $C_{i}$, we assume hereafter in the proof that $\bigcup_{j \in\left[\ell_{i}^{\prime}\right]} \mathcal{R}_{i, j}$ indexes the first $t_{i}$ coordinate 4 of $\mathrm{C}_{i}$. Letting $\mathrm{A}_{i}$ be an ambient space of $\mathrm{C}_{i}$, we denote by $A_{i}^{\prime}$ the set of the distinct $t_{i}$-prefixes of the vectors in $A_{i}$; the sequence $\left(\mathrm{A}_{i}^{\prime}\right)_{i=1}^{\infty}$ satisfies the conditions of Proposition 6.

Define

$$
w_{i}=\min \left\{\left\lfloor\frac{\theta}{2} \cdot t_{i}\right\rfloor,\left\lfloor\frac{d_{i}-1}{2}\right\rfloor\right\} .
$$

By (22)-23) (and (7)) we have:

$$
\lim _{i \rightarrow \infty} \frac{\omega_{i}}{t_{i}}=\frac{\theta}{2}
$$

${ }^{4}$ Namely, we deviate here from the notational convention that we set in Footnote 1
Next, we apply Lemma 10 with $C \leftarrow \mathrm{C}_{i}$ and $\mathrm{A} \leftarrow \mathrm{A}_{i}$, and with $\mathrm{B}$ taken as the set of all vectors in $\mathrm{A}_{i}$ whose $t_{i}$-prefixes are in $\mathrm{A}_{i}^{\prime}\left(w_{i} / t_{i}\right)$; namely,

$$
\mathrm{B}=\mathrm{A}_{i}^{\prime}\left(w_{i} / t_{i}\right) \times \mathrm{A}_{i}^{\prime \prime},
$$

where $\mathrm{A}_{i}^{\prime \prime}$ is the set of $\left(N_{i}-t_{i}\right)$-suffixes of the vectors of $\mathrm{A}_{i}$. It follows that there exists $\boldsymbol{y}=\left(\boldsymbol{y}^{\prime} \boldsymbol{y}^{\prime \prime}\right) \in \mathrm{A}_{i}$ (where $\boldsymbol{y}^{\prime} \in \mathrm{A}_{i}^{\prime}$ ) for which the intersection

$$
\mathrm{C}_{i}^{*}=\left(\boldsymbol{y}+\mathrm{C}_{i}\right) \cap \mathrm{B}
$$

satisfies

$$
\left|\mathrm{C}_{i}\right| \leq \frac{\left|\mathrm{A}_{i}\right| \cdot\left|\mathrm{C}_{i}^{*}\right|}{|\mathrm{B}|}=\frac{\left|\mathrm{A}_{i}^{\prime}\right|}{\left|\mathrm{A}_{i}^{\prime}\left(w_{i} / t_{i}\right)\right|} \cdot\left|\mathrm{C}_{i}^{*}\right| .
$$

Now, on the one hand, the code $-\boldsymbol{y}+\mathrm{C}_{i}^{*}$ is an all-disjoint linearly recoverable LRC with locality $r=n-1$ and minimum distance $\geq d_{i}$; yet, on the other hand, all the $t_{i}$-prefixes of its codewords are at distance at most $w_{i}$ from $-\boldsymbol{y}^{\prime}$, which means that any two prefixes are at most $2 w_{i}$ apart. It follows that all the $\left(N_{i}-t_{i}\right)$-suffixes of the codewords of $-\boldsymbol{y}+\mathrm{C}_{i}^{*}$ form an all-disjoint linearly recoverable LRC, $\mathrm{C}_{i}^{\prime \prime}$, of minimum distance $\geq d_{i}-2 w_{i}(>0)$ (in particular, these suffixes are all distinct). Hence,

$$
\left|C_{i}^{*}\right|=\left|C_{i}^{\prime \prime}\right| \leq M_{\text {LRC }}\left(N_{i}-t_{i}, d_{i}-2 w_{i}, n\right),
$$

where $M_{\mathrm{LRC}}(N, D, n)$ denotes the largest size of any alldisjoint linearly recoverable LRC over $F$ with length $N$, minimum distance $D$, and locality $n-1$. Combining (26) and (27) leads to

$$
\left|\mathrm{C}_{i}\right| \leq \frac{\left|\mathrm{A}_{i}^{\prime}\right|}{\left|\mathrm{A}_{i}^{\prime}\left(w_{i} / t_{i}\right)\right|} \cdot M_{\mathrm{LRC}}\left(N_{i}-t_{i}, d_{i}-2 w_{i}, n\right),
$$

and taking logarithms and dividing by $N_{i}$ yield the following upper bound on the rate, $R_{i}=\left(\log _{q}\left|C_{i}\right|\right) / N_{i}$, of $C_{i}$ :

$$
\begin{aligned}
R_{i} \leq & \frac{1}{N_{i}}\left(\log _{q} \frac{\left|\mathrm{A}_{i}^{\prime}\right|}{\left|\mathrm{A}_{i}^{\prime}\left(w_{i} / t_{i}\right)\right|}\right. \\
& \left.\quad+\log _{q} M_{\mathrm{LRC}}\left(N_{i}-t_{i}, d_{i}-2 w_{i}, n\right)\right) \\
= & \frac{t_{i}}{N_{i}} \cdot\left(\frac{1}{t_{i}} \log _{q} \frac{\left|\mathrm{A}_{i}^{\prime}\right|}{\left|\mathrm{A}_{i}^{\prime}\left(w_{i} / t_{i}\right)\right|}\right) \\
& +\left(1-\frac{t_{i}}{N_{i}}\right) \cdot \frac{\log _{q} M_{\mathrm{LRC}}\left(N_{i}-t_{i}, d_{i}-2 w_{i}, n\right)}{N_{i}-t_{i}} .
\end{aligned}
$$

We now take the limit as $i \rightarrow \infty$ of each of the terms in (28). By (24) and Proposition 6 we get

$$
\varlimsup_{i \rightarrow \infty} \frac{1}{t_{i}} \log _{q} \frac{\left|\mathrm{A}_{i}^{\prime}\right|}{\left|\mathrm{A}_{i}^{\prime}\left(w_{i} / t_{i}\right)\right|} \leq R_{0}\left(\frac{\theta}{2}, n\right) .
$$

In addition,

$$
\varliminf_{i \rightarrow \infty} \frac{d_{i}-2 w_{i}}{N_{i}-t_{i}} \stackrel{222-24]}{\geq} \frac{\delta-\tau \cdot \theta}{1-\tau} \stackrel{\underline{\underline{7}}}{=} \theta^{\prime}
$$

and, so,

$$
\varlimsup_{i \rightarrow \infty} \frac{\log _{q} M_{\mathrm{LRC}}\left(N_{i}-t_{i}, d_{i}-2 w_{i}, n\right)}{N_{i}-t_{i}} \leq R_{\mathrm{LRC}}\left(\theta^{\prime}, n\right) .
$$


We conclude that the rate $R$ of the code sequence $\left(\mathrm{C}_{i}\right)_{i=1}^{\infty}$ satisfies

$$
R=\varlimsup_{i \rightarrow \infty} R_{i} \leq \tau \cdot R_{0}\left(\frac{\theta}{2}\right)+(1-\tau) \cdot R_{\mathrm{LRC}}\left(\theta^{\prime}, n\right) .
$$

The sought result is reached by minimizing over $\theta$ and $\tau$.

We now turn to the proof of Theorem 2 Recall that the expression $R_{2}(\delta, n)$ therein involves the value $R_{\text {opt }}(\delta, \omega)$, being the supremum over all rates of sequences $\left(C_{i}\right)_{i=1}^{\infty}$ with r.m.d. $\geq \delta$ such that the codewords in each $C_{i}$ all have the same weight $\approx \omega N_{i}$. For our purposes, it would be convenient if this definition were relaxed so that the codeword weights only need to be bounded from above by (approximately) $\omega N_{i}$. It turns out that $R_{\mathrm{opt}}(\delta, \omega)$ is the rate supremum also under this relaxed setting, provided that $\omega \in[0,(q-1) / q]$. We make this statement precise in Lemma 11 below.

For $N, d, w \in \mathbb{Z}^{+}$, let $M_{\text {opt }}(N, d, w)$ denote the largest size of any code in $F^{N}$ with minimum distance $d$ and codeword weights that are all bounded from above by $w$. Define

$$
R_{\mathrm{opt}}(\delta, \leq \omega)=\sup _{\substack{d \in \mathcal{L}(\delta) \\ \boldsymbol{w} \in \mathcal{U}(\omega)}} \varlimsup_{N \rightarrow \infty} \frac{1}{N} \log _{q} M_{\mathrm{opt}}\left(N, d_{N}, w_{N}\right),
$$

where $\mathcal{L}(\delta)$ (respectively, $\mathcal{U}(\omega)$ ) is the set of all sequences $\left(a_{N}\right)_{N=1}^{\infty}$ over $\mathbb{Z}^{+}$satisfying $\underline{\lim }_{N \rightarrow \infty} a_{N} / N \geq \delta$ (respectively, $\left.\overline{\lim }_{N \rightarrow \infty} a_{N} / N \leq \omega\right)$.

We have the following technical lemma, which is proved in Appendix $\mathrm{A}$

Lemma 11. For every $\delta, \omega \in[0,(q-1) / q]$,

$$
R_{\text {opt }}(\delta, \leq \omega)=R_{\text {opt }}(\delta, \omega) .
$$

Proof of Theorem 2 Let $\left(\mathrm{C}_{i}\right)_{i=1}^{\infty}$ be an all-disjoint linearly recoverable $(\delta, n)$-LRC sequence over $F$. Letting $\mathrm{A}_{i}$ be an ambient space of $C_{i}$, the sequence $\left(A_{i}\right)_{i=1}^{\infty}$ satisfies the conditions of Proposition 6 ,

Fixing any $\omega \in[\delta / 2,(q-1) / q]$ and substituting $\mathrm{C} \leftarrow \mathrm{C}_{i}$, $\mathrm{A} \leftarrow \mathrm{A}_{i}$, and $\mathrm{B} \leftarrow \mathrm{A}_{i}(\omega)$ in Lemma 10 yield that there exists $\boldsymbol{y} \in \mathrm{A}_{i}$ for which

$$
\begin{aligned}
\left|\mathrm{C}_{i}\right| \cdot\left|\mathrm{A}_{i}(\omega)\right| & \leq\left|\mathrm{A}_{i}\right| \cdot\left|\left(\boldsymbol{y}+\mathrm{C}_{i}\right) \cap \mathrm{A}_{i}(\omega)\right| \\
& \leq\left|\mathrm{A}_{i}\right| \cdot M_{\mathrm{opt}}\left(N_{i}, d_{i}, \omega\right),
\end{aligned}
$$

where $N_{i}$ and $d_{i}$ are the length and the minimum distance of $\mathrm{C}_{i}$, respectively. Taking logarithms and dividing by $N_{i}$ yield the following upper bound on the rate $R_{i}$ of $\mathrm{C}_{i}$ :

$$
R_{i} \leq \frac{1}{N_{i}} \log _{q} \frac{\left|\mathrm{A}_{i}\right|}{\left|\mathrm{A}_{i}(\omega)\right|}+\frac{1}{N_{i}} \log _{q} M_{\mathrm{opt}}\left(N_{i}, d_{i}, w_{i}\right),
$$

where $w_{i}=\left\lfloor\omega N_{i}\right\rfloor$. The result is obtained by taking the limit as $i \rightarrow \infty$. Specifically, on the left-hand side of (30) we get the rate $R=\varlimsup_{i \rightarrow \infty} R_{i}$ of $\left(C_{i}\right)_{i=1}^{\infty}$, and on the right-hand side the respective terms become

$$
\varlimsup_{i \rightarrow \infty} \frac{1}{N_{i}} \log _{q} \frac{\left|\mathrm{A}_{i}\right|}{\left|\mathrm{A}_{i}(\omega)\right|} \stackrel{\text { Proposition } 6}{\leq} R_{0}(\omega, n)
$$

and

$$
\varlimsup_{i \rightarrow \infty} \frac{1}{N_{i}} \log _{q} M_{\mathrm{opt}}\left(N_{i}, d_{i}, w_{i}\right) \stackrel{\text { Lemma }}{\leq} R_{\mathrm{opt}}(\delta, \omega) .
$$

\section{THE CASE $q=2$ AND $n=3$}

We consider in this section the case $n=3$ over the binary field. We assume that the LRCs are all-disjoint, but not necessarily linearly recoverable.

As a warm-up, we start with the case $n=2$ over any finite field. It is straightforward to see that if an all-disjoint $(N, M, d)$ LRC $C$ over $F$ with locality $n-1=1$ has no fixed (in particular, trivial) coordinates, then all the repair groups have to be of size (exactly) 2 , and all the constituent codes have minimum distance (exactly) 25 Thus, both $N$ and $d$ are even and, without loss of generality, each constituent code is the $[2,1,2]$ repetition code over $F$. We can therefore view $C$ as a concatenated code over $F$ comprising an outer $(N / 2, M, d / 2)$ code over $F$ and an inner $[2,1,2]$ repetition code over $F$. We conclude that the rate of any all-disjoint $(\delta, 2)$-LRC sequence over $F$ is bounded from above by $(1 / 2) \cdot R_{\text {opt }}(\delta)$; moreover, we can get arbitrarily close to this bound by a concatenated code construction 6

We now turn to the case $n=3$ when $F=\mathbb{F}_{2}$. Let $C$ be an all-disjoint $(N, M, d)$ LRC over $F$ with locality $n-1=2$ and without fixed coordinates, and assume first that all the repair groups have size exactly 3 . Then each constituent code $(\mathrm{C})_{\mathcal{R}_{j}}$, being of length 3 and minimum distance $\geq 2$ and having no fixed coordinates, has size 2,3 , or 4 . It is rather easy to see that such codes are essentially unique (up to a replacement $0 \leftrightarrow 1$ at any given coordinate across all codewords). Moreover, any $(3,3,2)$ code over $F$ is necessarily equi-distant (i.e., the distance between any two distinct codewords is exactly 2) and can be augmented by a fourth codeword while still remaining equi-distant. Hence, we assume that each constituent code is either the $[3,1,3]$ repetition code or the $[3,2,2]$ parity code (which is equi-distant). We can therefore view $\mathrm{C}$ as a concatenated code over $F$ comprising an outer code $\mathbb{C}$ of length $\ell=N / 3$ and size $M$ over a mixed alphabet, namely:

$$
\mathbb{C} \subseteq F^{t} \times\left(F^{2}\right)^{\ell-t},
$$

for some $t \leq \ell$. The first $t$ (binary) coordinates are mapped to an inner code which is the $[3,1,3]$ repetition code, and the remaining $\ell-t$ (quaternary) coordinates are mapped to the $[3,2,2]$ parity code. Writing each codeword of $\mathbb{C}$ as $\left(\boldsymbol{c} \boldsymbol{c}^{\prime}\right)$, where $c \in F^{t}$ and $c^{\prime} \in\left(F^{2}\right)^{\ell-t}$, the following inequality must hold for any two distinct codewords $\left(\boldsymbol{c}_{1} \boldsymbol{c}_{1}^{\prime}\right),\left(\boldsymbol{c}_{2} \boldsymbol{c}_{2}^{\prime}\right) \in \mathbb{C}$ to maintain the minimum distance $d$ of $\mathrm{C}$ :

$$
3 \mathrm{~d}_{F}\left(\boldsymbol{c}_{1}, \boldsymbol{c}_{2}\right)+2 \mathrm{~d}_{F^{2}}\left(\boldsymbol{c}_{1}^{\prime}, \boldsymbol{c}_{2}^{\prime}\right) \geq d,
$$

where $\mathrm{d}_{Q}(\cdot, \cdot)$ denotes Hamming distance over the alphabet $Q$. Regarding now $\mathrm{C}$ as an element in a $(\delta, 3)$-LRC sequence where $t / \ell$ converges to $\tau \in[0,1]$, the condition $(31)$ becomes

$$
\tau \cdot \frac{\mathrm{d}_{F}\left(\boldsymbol{c}_{1}, \boldsymbol{c}_{2}\right)}{t}+\frac{2}{3}(1-\tau) \cdot \frac{\mathrm{d}_{F^{2}}\left(\boldsymbol{c}_{1}^{\prime}, \boldsymbol{c}_{2}^{\prime}\right)}{\ell-t} \geq \delta .
$$

${ }^{5}$ If $\mathrm{C}$ does contain trivial coordinates, we can shorten it on these coordinates, thereby only increasing the r.m.d. and the rate and, thus, obtaining an upper bound on that larger rate.

${ }^{6}$ This upper bound holds in fact also when the repair groups are not necessarily disjoint, as overlapping repair groups force all the entries that are indexed by their union to be equal. This corresponds to having inner repetition codes of rate smaller than $1 / 2$. 
We are interested in finding the value of $\tau$ for which the rate of the LRC sequence is maximized, subject to satisfying (32). We do this using the following lemma; the function $\delta \mapsto R_{\mathrm{LP} ; Q}(\delta)$ stands for the (second) linear-programming bound, due to Aaltonen [1, p. 141], on the rate of code sequences over a finite alphabet $Q$ (see also [4, Theorem 1]).

Lemma 12. Let $Q$ and $Q^{\prime}$ be finite alphabets and fix $\beta, \beta^{\prime} \in$ $\mathbb{R}^{+}$and $\tau \in[0,1]$. Let $\left(\mathbb{C}_{i}\right)_{i=1}^{\infty}$ be a code sequence such that

$$
\mathbb{C}_{i} \subseteq Q^{t_{i}} \times\left(Q^{\prime}\right)^{\ell_{i}-t_{i}}
$$

where $\lim _{i \rightarrow \infty} t_{i} / \ell_{i}=\tau$. Suppose also that the (weighted) r.m.d. of the sequence is at least $\delta$, where, for the purpose of computing distances, each coordinate of $Q$ (respectively, $Q^{\prime}$ ) contributes $\beta$ (respectively, $\beta^{\prime}$ ) to the distance. Then

$$
\begin{aligned}
\varlimsup_{i \rightarrow \infty} \frac{\log \left|\mathbb{C}_{i}\right|}{\ell_{i}} \leq \min _{\left(\theta, \theta^{\prime}\right)}\left\{\tau \cdot R_{\mathrm{LP} ; Q}(\theta) \log |Q|\right. \\
\left.+(1-\tau) \cdot R_{\mathrm{LP} ; Q^{\prime}}\left(\theta^{\prime}\right) \log \left|Q^{\prime}\right|\right\},
\end{aligned}
$$

where the minimum is taken over $\left(\theta, \theta^{\prime}\right) \in[0,1]^{2}$ such that

$$
\tau \cdot \beta \cdot \theta+(1-\tau) \cdot \beta^{\prime} \cdot \theta^{\prime}=\delta .
$$

A special case of this lemma, for the case $Q=Q^{\prime}$ and $\beta=\beta^{\prime}$, was proved by Ben-Haim and Litsyn in [4] 7 For this case, Lemma 12 states that the bound $\delta \mapsto R_{\mathrm{LP} ; Q}(\delta)$ can be improved by taking its lower convex envelope (it turns out that this bound is not convex for general $Q$ ). With little effort, Theorem 5 in [4] can be adapted to the case of mixed alphabets and weighted distance $[5] .8$

Applying Lemma 12 with $Q=\mathbb{F}_{2}, Q^{\prime}=\mathbb{F}_{4}, \beta=1$, and $\beta^{\prime}=2 / 3$, we have verified numerically that the expression (33) is maximized when $\tau=0$. Hence, we get the following upper bound on the rate $R$ of any all-disjoint $(\delta, 3)$ LRC sequence over $F$ in which the repair groups are all of size 3:

$$
R \leq \frac{2}{3} \cdot R_{\mathrm{LP} ; \mathbb{F}_{4}}\left(\frac{3 \delta}{2}\right) .
$$

It remains to consider the case where the $(N, M, d)$ LRC $\mathrm{C}$ has repair groups of size 2, namely, some $s$ out of the $t$ coordinates of the outer code $\mathbb{C}\left(\subseteq F^{t} \times\left(F^{2}\right)^{\ell-t}\right)$ map to the $[2,1,2]$ repetition code. By adding $s$ information bits to $\mathbb{C}$ (thereby increasing its size by a factor of $2^{s}$ ) we can obtain a new concatenated code $C^{*}$ in which we replace all the inner instances of the $[2,1,2]$ repetition code by instances of the $[3,2,2]$ parity code. Doing so, the overall code length becomes $N+s$, the minimum distance remains unchanged (and, so, the r.m.d. reduces by a factor of $N /(N+s)$ ), and the rate becomes

$$
\frac{\left(\log _{2} M\right)+s}{N+s}=\frac{\left(\log _{2} M\right) / N+(s / N)}{1+(s / N)} .
$$

\footnotetext{
${ }^{7}$ This is not stated so explicitly in [4], but this is what Theorem 7 in 4 reduces to when Eq. (36) in that paper is plugged into Eq. (39) and the result is then plugged into Eq. (40).

${ }^{8}$ Specifically, one only needs to modify the definition of the function $f$ in that theorem so that the first multiplicand therein is $\beta\left(a_{1}+b_{1}-x_{1}-\right.$ $\left.y_{1}\right)+\beta^{\prime}\left(a_{2}+b_{2}-x_{2}-y_{2}\right)$. The proof, as is, holds also when the prefixes and suffixes - of lengths $n_{1}$ and $n_{2}$ - of the codewords are over different alphabets.
}

Switching to a code sequence $\left(\mathrm{C}_{i}\right)_{i=1}^{\infty}$ where $\lim _{i \rightarrow \infty} s_{i} / N_{i}=$ $\sigma$, we get from (35) the following relationship between the rate $R$ of the sequence and the rate $R^{*}$ of $\left(\mathrm{C}_{i}^{*}\right)_{i=1}^{\infty}$ :

$$
R^{*}=\frac{R+\sigma}{1+\sigma}
$$

On the other hand, the bound (34) applies to $\left(\mathrm{C}_{i}^{*}\right)_{i=1}^{\infty}$, namely:

$$
R^{*} \leq \frac{2}{3} \cdot R_{\mathrm{LP} ; \mathbb{F}_{4}}\left(\frac{3 \delta}{2(1+\sigma)}\right) .
$$

Combining the last two equations yields:

$$
R \leq \frac{2(1+\sigma)}{3} \cdot R_{\mathrm{LP} ; \mathbb{F}_{4}}\left(\frac{3 \delta}{2(1+\sigma)}\right)-\sigma .
$$

We have verified numerically that this expression is maximized when $\sigma=0$. We therefore conclude that (34) holds for any all-disjoint $(\delta, 3)$-LRC sequence over $F$. The bound (34) is depicted in Figure 2 (curve $(\mathrm{g})$ ).

\section{THE GENERAL LINEARLY RECOVERABLE CASE}

In this section, we present some (weaker) bounds for $(\delta, n)$ LRC sequences that are not necessarily all-disjoint (but they are still linearly recoverable). Our results are based on deriving the asymptotic version of the sphere-packing bound of [28] (Theorems 5 and 12 therein).

Let $\mathrm{C}$ be a linearly recoverable LRC of length $N$, minimum distance $d$, and locality $r=n-1$ over $F$ and let $\left(\mathcal{R}_{j}\right)_{j \in[N]}$ be a list of repair groups of $C$. We assume hereafter in this section (without loss of generality) that this list satisfies the following conditions.

R1) $\left|\mathcal{R}_{j}\right| \leq n$ for each index $j \in[N]$ (this condition follows directly from the locality).

R2) For each $j \in[N]$, the repair group $\mathcal{R}_{j}$ is minimal in the sense that no proper subset of it is a repair group for $j$; this, in turn, implies that $\mathcal{R}_{j}$ is a repair group for all $j^{\prime} \in \mathcal{R}_{j}$.

R3) Each repair group $\mathcal{R}_{j}$ contains at least one index $j^{\prime}$ that is not contained in any repair group $\mathcal{R}_{i} \neq \mathcal{R}_{j}$ (otherwise, $\mathcal{R}_{j}$ can be spared).

Considering now only the set of distinct repair groups $\left\{\mathcal{R}_{j}\right\}_{j \in[\ell]}$ of $\mathrm{C}$, for each repair group $\mathcal{R}_{j}$, the constituent code $(\mathrm{C})_{\mathcal{R}_{j}}$ is contained in a linear $\left[\left|\mathcal{R}_{j}\right|,\left|\mathcal{R}_{j}\right|-1\right]$ code over $F$, which we denote by $\mathcal{C}_{j}$; moreover, by condition (R2), the code $\mathcal{C}_{j}$ has minimum distance 2 . An ambient space $\mathrm{A}$ of $\mathrm{C}$ is defined as in 21); by condition (R3), the containment in 21) holds in fact with equality for all $j \in[\ell]$.

The method of [28] for obtaining a (non-asymptotic) spherepacking-type bound on the rate of $\mathrm{C}$ is based on shortening $\mathrm{C}$ on the set of coordinates, $\mathcal{S}$, on which repair groups intersect, thereby reducing to the all-disjoint case. Denoting $\nu=|\mathcal{S}| / N$, the resulting shortened code, $\widehat{\mathrm{C}}$, is an all-disjoint linearly recoverable LRC of length $(1-\nu) N$ and minimum distance $\geq d$, with the following $\ell$ distinct repair groups:

$$
\widehat{\mathcal{R}}_{j}=\mathcal{R}_{j} \backslash \mathcal{S}, \quad j \in[\ell] .
$$


Now, on the one hand, we have:

$$
\begin{aligned}
\ell \cdot n & \geq \sum_{j \in[\ell]}\left|\mathcal{R}_{j}\right|=\sum_{t \in[N]}\left|\left\{j \in[\ell]: t \in \mathcal{R}_{j}\right\}\right| \\
& =\sum_{t \in \mathcal{S}}\left|\left\{j: t \in \mathcal{R}_{j}\right\}\right|+\sum_{t \in[N] \backslash \mathcal{S}}\left|\left\{j: t \in \mathcal{R}_{j}\right\}\right| \\
& \geq 2|\mathcal{S}|+(N-|\mathcal{S}|) .
\end{aligned}
$$

On the other hand, by condition (R3), we also have:

$$
N-|\mathcal{S}| \geq \ell \text {. }
$$

From (36) and 37) we get:

$$
\frac{1+\nu}{n} \leq \frac{\ell}{N} \leq 1-\nu
$$

and, in particular,

$$
0 \leq \nu \leq \frac{n-1}{n+1}
$$

Moreover, from 38 we get that the average size of the distinct (and disjoint) repair groups of $\widehat{C}$ satisfies

$$
\frac{1}{\ell} \sum_{j \in[\ell]}\left|\widehat{\mathcal{R}}_{j}\right|=\frac{(1-\nu) N}{\ell} \leq \frac{1-\nu}{1+\nu} \cdot n=\mu .
$$

The rate $R=\left(\log _{q}|\mathrm{C}|\right) / N$ of $\mathrm{C}$ is related to that of $\widehat{\mathrm{C}}$ by:

$$
R=\frac{1}{N} \cdot \log _{q}|\mathrm{C}| \leq \frac{1}{N}\left(|\mathcal{S}|+\log _{q}|\widehat{\mathrm{C}}|\right)=\nu+(1-\nu) \cdot \widehat{R}
$$

Thus, given $\nu$, any upper bound on $\widehat{R}$ implies an upper bound on $R$, and the dependence on $\nu$ can then be eliminated by maximizing the latter bound over $\nu \in[0,(n-1) /(n+1)]$.

Using the above strategy, we next turn to adapting our previous bounds to linearly recoverable $(\delta, n)$-LRC sequences that are not necessarily all-disjoint. We will make use of the following notation. For $n \in \mathbb{R}^{+}$and $\omega \in \mathbb{R}_{\geq 0}$, define:

$$
\widehat{R}_{0}(\omega, n)=\max _{\nu}\left\{\nu+(1-\nu) \cdot \bar{R}_{0}\left(\frac{\omega}{1-\nu}, n, \frac{1-\nu}{1+\nu} \cdot n\right)\right\},
$$

where $\bar{R}_{0}(\cdot, \cdot, \cdot)$ is as defined in (15) and the maximum is taken over all $\nu \in[0,(n-1) /(n+1)]$.

Remark 6. Substituting $\nu=0$ and $\nu=(n-1) /(n+1)$ in the objective function in 41, we get the following lower bound on $\widehat{R}_{0}(\omega, n)$ :

$$
\widehat{R}_{0}(\omega, n) \geq \max \left\{R_{0}(\omega, n), \frac{n-1}{n+1}\right\} .
$$

Based on our numerical evidence, we conjecture that this lower bound is tight (see also Remark 10 in Appendix B).

The following proposition is a (weaker) counterpart of Proposition 6 for the case where repair groups can intersect.

Proposition 13. Given $n \in \mathbb{Z}^{+}$, let $\left(\mathrm{A}_{i}\right)_{i=1}^{\infty}$ be an infinite sequence of codes over $F$ where each $\mathrm{A}_{i}$ is a linear code of length $N_{i}$ over $F$ defined by

$$
\left(\mathrm{A}_{i}\right)_{\mathcal{R}_{i, j}}=\mathcal{C}_{i, j}, \quad j \in\left[N_{i}\right],
$$

with the list $\left(\mathcal{R}_{i, j}\right)_{j \in\left[N_{i}\right]}$ satisfying conditions $(R 1)-(R 3)$ and each constituent code $\mathcal{C}_{i, j}$ being a linear $\left[\left|\mathcal{R}_{i, j}\right|,\left|\mathcal{R}_{i, j}\right|-1,2\right]$ code over $F$. Then for any nonnegative real sequence $\left(\omega_{i}\right)_{i=1}^{\infty}$ such that $\underline{\lim }_{i \rightarrow \infty} \omega_{i}=\omega$ :

$$
\varlimsup_{i \rightarrow \infty} \frac{1}{N_{i}} \log _{q} \frac{\left|\mathrm{A}_{i}\right|}{\left|\mathrm{A}_{i}\left(\omega_{i}\right)\right|} \leq \widehat{R}_{0}(\omega, n) .
$$

Proof. For each $i \in \mathbb{Z}^{+}$, let $\widehat{\mathrm{A}}_{i}$ be obtained by shortening $\mathrm{A}_{i}$ on the set of coordinates on which repair groups (i.e., subsets) $\mathcal{R}_{i, j}$ intersect. Denoting by $\nu_{i}(\in[0,(n-1) /(n+1)])$ the fraction of removed coordinates, by possibly restricting to a subsequence of the codes, we can assume that $\left(\nu_{i}\right)_{i=1}^{\infty}$ converges to a limit $\nu$ and that the length, $m_{i}=\left(1-\nu_{i}\right) N_{i}$, of $\widehat{\mathrm{A}}_{i}$ strictly increases with $i$. We then get that the code sequence $\left(\widehat{A}_{i}\right)_{i=1}^{\infty}$ satisfies the conditions of Proposition 6 (and, hence, of Lemma 8); in particular, since each $\mathcal{C}_{i, j}$ has minimum distance 2 , the respective (shortened) constituent codes of each $\widehat{A}_{i}$ all (still) have redundancy 1 . Moreover, the average length of those constituent codes is bounded from above by

$$
\frac{1-\nu_{i}}{1-\nu_{i}} \cdot n
$$

(as in (39). In addition,

$$
\frac{1}{N_{i}} \log _{q}\left|\mathrm{~A}_{i}\right| \leq \nu_{i}+\left(1-\nu_{i}\right) \cdot \frac{1}{m_{i}} \log _{q}\left|\widehat{\mathrm{A}}_{i}\right|
$$

(as in (40) and, for any nonnegative real sequence $\left(\omega_{i}\right)_{i=1}^{\infty}$ :

$$
\begin{array}{r}
\frac{1}{N_{i}} \log _{q}\left|\mathrm{~A}_{i}\left(\omega_{i}\right)\right| \geq \frac{1}{N_{i}} \log _{q}\left|\widehat{\mathrm{A}}_{i}\left(\omega_{i} /\left(1-\nu_{i}\right)\right)\right| \\
=\left(1-\nu_{i}\right) \cdot \frac{1}{m_{i}} \log _{q}\left|\widehat{\mathrm{A}}_{i}\left(\omega_{i} /\left(1-\nu_{i}\right)\right)\right| .
\end{array}
$$

Therefore,

$$
\begin{aligned}
& \varlimsup_{i \rightarrow \infty} \frac{1}{N_{i}} \log _{q} \frac{\left|\mathrm{A}_{i}\right|}{\left|\mathrm{A}_{i}\left(\omega_{i}\right)\right|} \\
& \stackrel{422+43}{\leq} \nu+(1-\nu) \cdot \varlimsup_{i \rightarrow \infty} \frac{1}{m_{i}} \log _{q} \frac{\left|\widehat{\mathrm{A}}_{i}\right|}{\left|\widehat{\mathrm{A}}_{i}\left(\omega_{i} /\left(1-\nu_{i}\right)\right)\right|} \\
& \stackrel{\text { Lemma } 8}{\leq} \nu+(1-\nu) \cdot \bar{R}_{0}\left(\frac{\omega}{1-\nu}, n, \frac{1-\nu}{1+\nu} n\right) \\
& \leq \widehat{R}_{0}(\omega, n),
\end{aligned}
$$

where $\omega=\underline{\lim }_{i \rightarrow \infty} \omega_{i}$.

The following sphere-packing bound is proved similarly to Theorem 9, except that we use Proposition 13 instead of Proposition 6 .

Theorem 14. The rate of any linearly recoverable $(\delta, n)$ LRC sequence over $F$ is bounded from above by

$$
\widehat{R}_{\mathrm{SP}}(\delta, n)=\widehat{R}_{0}\left(\frac{\delta}{2}, n\right) .
$$

Next are our (weaker) versions of Theorems 1 and 2 for general linearly recoverable LRC sequences.

Theorem 15. The rate of any linearly recoverable $(\delta, n)$ LRC sequence over $F$ is bounded from above by

$$
\begin{aligned}
& \widehat{R}_{1}(\delta, n)=\inf _{\tau \in(0,1)} \min _{\left(\theta, \theta^{\prime}\right)}\left\{\tau \cdot \widehat{R}_{0}\left(\frac{\theta}{2}, n\right)\right. \\
& \left.+(1-\tau) \cdot R_{\mathrm{opt}}\left(\theta^{\prime}, n\right)\right\} \text {, }
\end{aligned}
$$


where the (inner) minimum is taken over all pairs $\left(\theta, \theta^{\prime}\right)$ in $[0,(q-1) / q]^{2}$ that satisfy (7).

Proof. The proof resembles that of Theorem 1, yet requires several modifications which are described below. Given a linearly recoverable $(\delta, n)$-LRC sequence $\left(C_{i}\right)_{i=1}^{\infty}$ over $F$, we let $\left(\mathcal{R}_{i, j}\right)_{j \in\left[N_{i}\right]}$ be a list of repair groups of $\mathrm{C}_{i}$ that satisfies conditions (R1)-(R3). Letting $\left\{\mathcal{R}_{i, j}\right\}_{j \in\left[\ell_{i}\right]}$ be the set of distinct repair groups in the list, we define (as in the proof of Theorem 1)

$$
t_{i}=\left|\bigcup_{j \in\left[\ell_{i}^{\prime}\right]} \mathcal{R}_{i, j}\right|,
$$

where $\ell_{i}^{\prime}$ is the smallest in $\left[\ell_{i}\right]$ so that $t_{i} \geq \tau N_{i}$. And by possibly permuting the coordinates of $\mathrm{C}_{i}$, we assume that $\bigcup_{j \in\left[\ell_{i}^{\prime}\right]} \mathcal{R}_{i, j}=\left[t_{i}\right]$.

We now specify the changes to the proof of Theorem 1 First, we need to modify the argument that leads to Eq. 26), since the decomposition (25) may no longer hold. Still, due to condition (R3), the size of the following set is the same for all $\boldsymbol{y}^{\prime} \in \mathrm{A}_{i}^{\prime}$ :

$$
\left\{\boldsymbol{y}^{\prime \prime} \in F^{N_{i}-t_{i}}:\left(\boldsymbol{y}^{\prime} \boldsymbol{y}^{\prime \prime}\right) \in \mathrm{A}_{i}\right\} .
$$

Thus,

$$
\frac{\left|\mathrm{A}_{i}\right|}{|\mathrm{B}|}=\frac{\left|\mathrm{A}_{i}^{\prime}\right|}{\left|\mathrm{A}_{i}^{\prime}\left(w_{i} / t_{i}\right)\right|},
$$

which justifies (26).

Secondly, we need to weaken Eq. 277, since the code $C_{i}^{\prime \prime}$ is now not necessarily an LRC. Specifically, 27) now becomes

$$
\left|C_{i}^{*}\right|=\left|C_{i}^{\prime \prime}\right| \leq M_{\text {opt }}\left(N_{i}-t_{i}, d_{i}-2 w_{i}\right),
$$

where $M_{\mathrm{opt}}(N, D)$ denotes the largest size of any code over $F$ with length $N$ and minimum distance $D$. Accordingly, from this point in the proof of Theorem 1 we change the instances of $R_{\mathrm{LRC}}(\cdot, n)$ into $R_{\mathrm{opt}}(\cdot)$.

Finally, using Proposition 13, we change (29) into

$$
\varlimsup_{i \rightarrow \infty} \frac{1}{t_{i}} \log _{q} \frac{\left|\mathrm{A}_{i}^{\prime}\right|}{\left|\mathrm{A}_{i}^{\prime}\left(w_{i} / t_{i}\right)\right|} \leq \widehat{R}_{0}\left(\frac{\theta}{2}, n\right) .
$$

Below is our variant of Theorem 2 which is proved using Proposition 13 instead of Proposition 6

Theorem 16. The rate of any linearly recoverable $(\delta, n)$ $L R C$ sequence over $F$ is bounded from above by

$$
\widehat{R}_{2}(\delta, n)=\min _{\omega \in[\delta / 2,(q-1) / q]}\left\{\widehat{R}_{0}(\omega, n)+R_{\mathrm{opt}}(\delta, \omega)\right\} .
$$

Notice that unlike the all-disjoint case, we cannot substitute $\widehat{R}_{2}\left(\theta^{\prime}, n\right)$ for $R_{\text {opt }}\left(\theta^{\prime}, n\right)$ in Theorem 15

The various bounds are plotted in Figure 4 for $q=2$ and $n=4$. Curve (a) is the sphere-packing bound of Theorem 14 Curve (b) is identical to its counterpart in Figure 3 and, as it turns out, curve (c) is the same as in that figure too (namely, $\widehat{R}_{1}(\delta, n)=R_{1}(\delta, n)$ for the examined parameters); this is due to the fact that the minimum in Theorem 16 is attained at values $\theta$ where $\widehat{R}_{0}(\theta, n)=R_{0}(\theta, n)$. When plotting curve (d), we have taken the minimum of $R_{2}(\delta, n)$ and $R_{\mathrm{LP}}(\delta, n)$. Some values of the bounds are listed in Table [I] where entries that differ from those in Table $\prod$ are marked in italics. There is still a range where Theorem 16 yields the best upper bound, yet this range is smaller compared to the all-disjoint case.

Remark 7. A second variant of Theorem 2 can be obtained by first shortening the codes in a given LRC sequence on the intersections of repair groups, and then applying Theorem 2 to the resulting (all-disjoint) LRC sequence. This yields the upper bound

$$
\begin{aligned}
\widehat{R}_{3}(\delta, n)=\max _{\nu}\left\{\nu+(1-\nu) \cdot \min _{\omega}\{\right. & \bar{R}_{0}\left(\omega, n, \frac{1+\nu}{1-\nu} \cdot n\right) \\
& \left.\left.+R_{\mathrm{opt}}\left(\frac{\delta}{1-\nu}, \omega\right)\right\}\right\},
\end{aligned}
$$

where the outer maximum is over $\nu \in[0,(n-1) /(n+1)]$ and the inner minimum is over $\omega \in[\delta /(2-2 \nu),(q-1) / q]$. Yet at least for the parameters that we have tested, we have observed no difference between the values of $\widehat{R}_{2}(\delta, n)$ and $\widehat{R}_{3}(\delta, n)$.

TABLE II

VALUES OF THE BOUNDS FOR $q=2$ AND $n=4$ WITHOUT THE ALL-DISJOINT CONSTRAINT.

\begin{tabular}{ccccc}
\hline \hline$\delta$ & $(\mathrm{a})$ & $(\mathrm{b})$ & $(\mathrm{c})$ & $(\mathrm{d})$ \\
\hline 0.07 & 0.6133 & 0.6317 & 0.6131 & 0.6079 \\
0.10 & 0.6000 & 0.5809 & 0.5643 & 0.6000 \\
0.15 & 0.6000 & 0.4964 & 0.4830 & 0.6000 \\
0.30 & 0.6000 & 0.2427 & 0.2391 & 0.6000 \\
\hline \hline
\end{tabular}

\section{ACKNOWLEDGMENT}

I would like to thank Yael Ben-Haim and Simon Litsyn for helpful discussions and for making me aware of the results of [6].

\section{APPENDIX A \\ SKIPPED PROOFS}

Proof of Lemma 4 By convexity, for all $z \in(0,1]$ we have $\mathbb{E}\left\{z^{X}\right\} \geq z^{\mathbb{E}\{X\}}$, with equality holding when $z=1$; hence, $\gamma(u)=1$ when $u \geq \mathbb{E}\{X\}$. When $u \leq x_{\min }$, the infimum in (9) is attained at $z \rightarrow 0$ and, so, $\gamma(u)=0$ when $u<x_{\min }$ and $\gamma(u)=p\left(x_{\min }\right)$ when $u=x_{\min }$. For $x_{\min }<u<\mathbb{E}\{X\}$, we differentiate $g_{u}(z)$ with respect to $z$ to obtain

$$
g_{u}^{\prime}(z)=z^{x_{\min }-u-1} \cdot f_{u}(z),
$$

where

$$
f_{u}(z)=\sum_{x \in \mathcal{X}}(x-u) \cdot p(x) \cdot z^{x-x_{\min }} .
$$

Thus, $f_{u}(1)=\mathbb{E}\{X\}-u>0$ and $f_{u}(0)=\left(x_{\min }-u\right)$. $p\left(x_{\min }\right)<0$, which implies that the infimum in (9) is a proper minimum attained at an (interior) point $z_{u} \in(0,1)$; the point $z_{u}$ satisfies

$$
1 \geq g_{u}\left(z_{u}\right)>z_{u}^{x_{\min }-u} \cdot p\left(x_{\min }\right)
$$

i.e.,

$$
z_{u}>p\left(x_{\min }\right)^{1 /\left(u-x_{\min }\right)} .
$$


This means that $z_{u}$ is bounded away from zero whenever $u$ is bounded away from $x_{\min }$. Defining $z_{u}=1$ for $u \geq \mathbb{E}\{X\}$, for any $v>u \geq x_{\min }$ we have

$$
\gamma(u) \leq g_{u}\left(z_{v}\right) \leq z_{v}^{u-v} \cdot g_{u}\left(z_{v}\right)=g_{v}\left(z_{v}\right)=\gamma(v),
$$

with the second inequality being strict when $v<\mathbb{E}\{X\}$. Hence, $u \mapsto \gamma(u)$ is strictly increasing when $x_{\min } \leq u<$ $\mathbb{E}\{X\}$. On the other hand, we also have

$$
\gamma(u)=g_{u}\left(z_{u}\right)=z_{u}^{v-u} g_{v}\left(z_{u}\right) \geq z_{u}^{v-u} \gamma(v),
$$

which, combined with $\gamma(u) \leq \gamma(v)$, means that $u \mapsto \gamma(u)$ is continuous when $u>x_{\min }$. Moreover, it is right-continuous at $u=x_{\min }$, since

$$
\lim _{\varepsilon \rightarrow 0^{+}} \gamma\left(x_{\min }+\varepsilon\right) \leq \lim _{\varepsilon \rightarrow 0^{+}} g_{x_{\min }+\varepsilon}(\varepsilon)=p\left(x_{\min }\right)=\gamma\left(x_{\min }\right) .
$$

Finally, the concavity of $u \mapsto \log \gamma(u)$ follows from 111 and

$$
\begin{aligned}
& \text { Prob }\left\{\frac{1}{\ell_{1}+\ell_{2}} \sum_{i=1}^{\ell_{1}+\ell_{2}} X_{i} \leq \frac{\ell_{1}}{\ell_{1}+\ell_{2}} u_{1}+\frac{\ell_{2}}{\ell_{1}+\ell_{2}} u_{2}\right\} \\
& \geq \operatorname{Prob}\left\{\frac{1}{\ell_{1}} \sum_{i=1}^{\ell_{1}} X_{i} \leq u_{1}\right\} \cdot \operatorname{Prob}\left\{\frac{1}{\ell_{2}} \sum_{i=\ell_{1}+1}^{\ell_{1}+\ell_{2}} X_{i} \leq u_{2}\right\} .
\end{aligned}
$$

Turning to the proof of Lemma 11, it makes use of the following theorem, which was proved in [6, Theorem 1] for the special case of the binary alphabet. For completeness, we provide a proof of the theorem for general $q$ right after the proof of Lemma 11

Theorem 17. For any $\delta \in[0,(q-1) / q]$, the mapping $\omega \mapsto$ $R_{\mathrm{opt}}(\delta, \omega)$ is non-decreasing on $\omega \in[0,(q-1) / q]$.

Proof of Lemma 11] Obviously, $R_{\mathrm{opt}}(\delta, \omega) \leq R_{\mathrm{opt}}(\delta, \leq \omega)$. To prove the inequality in the other direction, Let $\left(C_{i}\right)_{i=1}^{\infty}$ be a code sequence with r.m.d. $\geq \delta$, with the (length- $N_{i}$ ) codewords of each $\mathrm{C}_{i}$ all having weight at most $w_{i}$, such that $\varlimsup_{i \rightarrow \infty} w_{i} / N_{i} \leq \omega$. Letting $\mathrm{C}_{i}^{*}$ be a largest constantweight subcode of $\mathrm{C}_{i}$ (of codeword weight $w_{i}^{*} \leq w_{i}$ ), we have $\left|C_{i}^{*}\right| \geq\left|C_{i}\right| /\left(w_{i}+1\right)$ and $\varlimsup_{i \rightarrow \infty} w_{i}^{*} / N_{i}=\omega^{*} \leq \omega$ (and by possibly restricting to a subsequence of $\left(\mathrm{C}_{i}^{*}\right)_{i=1}^{\infty}$ we can assume that $\omega^{*}$ is a proper limit of $\left.\left(w_{i} / N_{i}\right)_{i=1}^{\infty}\right)$. Thus,

$$
R_{\mathrm{opt}}(\delta, \leq \omega) \leq \sup _{\omega^{*} \in[0, \omega]} R_{\mathrm{opt}}\left(\delta, \omega^{*}\right)
$$

By Theorem 17 we then get that when $\omega \in[0,(q-1) / q]$, the supremum is attained at $\omega^{*}=\omega$.

Proof of Theorem 17 Given an Abelian group $F$ of size $q$, fix $\omega, \theta \in[0,(q-1) / q]$ and let $\mathcal{C}$ be a constant-weight code of length $N$ and minimum distance $d$ over $F$ with codeword weight $\lfloor\omega N\rfloor$. We show that there exists $\boldsymbol{y} \in F^{N}$ such that the translation $\boldsymbol{y}+\mathcal{C}$ contains a constant-weight subcode $\mathcal{C}^{*}$ of size

$$
\left|\mathcal{C}^{*}\right| \geq \frac{|\mathcal{C}|}{(N+1)^{3}}
$$

and of codeword weight $\left\lfloor\omega^{*} N\right\rfloor$, where

$$
\omega^{*}=\omega+\theta\left(1-\frac{q w}{q-1}\right) .
$$

The result will follow by observing that $\omega^{*}$ ranges over $[\omega,(q-1) / q]$ as $\theta$ ranges over $[0,(q-1) / q]$. Hereafter in the proof, we assume that $\omega$ and $\theta$ are rational numbers and $N$ is such that $\omega N, \theta N$, and $\theta \omega N /(q-1)$ are integers (it is easy to see that those assumptions are allowed in order to obtain the asymptotic result that we seek).

For any codeword $c \in \mathcal{C}$, let $\mathcal{T}(\boldsymbol{c})$ denote the set of all words $\boldsymbol{y} \in F^{N}$ that satisfy the following conditions.

Y1) The subword $\boldsymbol{y}^{\prime} \in F^{\omega N}$ of $\boldsymbol{y}$ that is indexed by the support of $\boldsymbol{c}$ has weight $\theta \omega N$,

Y2) a fraction $1 /(q-1)$ of the nonzero entries of $-\boldsymbol{y}^{\prime}$ agree with the respective entries in $c$, and-

Y3) the subword $\boldsymbol{y}^{\prime \prime} \in F^{(1-\omega) N}$ of $\boldsymbol{y}$ that is indexed by the zero entries of $\boldsymbol{c}$ has weight $\theta(1-\omega) N$.

It follows from these conditions that the weight of each $\boldsymbol{y} \in \mathcal{T}(\boldsymbol{c})$ is $\theta N$ and that for each $\boldsymbol{y} \in \mathcal{T}(\boldsymbol{c})$, the weight of $\boldsymbol{y}+\boldsymbol{c}$ is

$$
\left(\frac{q-2}{q-1} \cdot \theta \omega+(1-\theta) \omega+\theta(1-\omega)\right) N \stackrel{45}{=} \omega^{*} N .
$$

We also have:

$$
\begin{aligned}
|\mathcal{T}(\boldsymbol{c})|=\left(\begin{array}{c}
\omega N \\
\theta \omega N
\end{array}\right) & \\
& \cdot\left(\begin{array}{c}
\theta \omega N \\
\theta \omega N /(q-1)
\end{array}\right) \cdot(q-2)^{\theta \omega N(q-2) /(q-1)} \\
& \cdot\left(\begin{array}{c}
(1-\omega) N \\
\theta(1-\omega) N
\end{array}\right) \cdot(q-1)^{\theta(1-\omega) N},
\end{aligned}
$$

where the first term is the number of possible supports of a subword $\boldsymbol{y}^{\prime}$ that satisfies condition (Y1) and, for each such support, the second term counts the number of subwords $\boldsymbol{y}^{\prime}$ that satisfy condition (Y2). The third term counts the number of subwords $\boldsymbol{y}^{\prime \prime}$ that satisfy condition (Y3).

We now apply the following well known approximation of the binomial coefficients:

$$
\mathrm{H}\left(\frac{k}{m}\right)-\frac{\log (m+1)}{m} \leq \frac{1}{m} \log \left(\begin{array}{c}
m \\
k
\end{array}\right) \leq \mathrm{H}\left(\frac{k}{m}\right),
$$

where $x \mapsto \mathrm{H}(x)$ is the (binary) entropy function $-x \log x-$ $(1-x) \log (1-x)$ (see [23, pp. 105-106]). We get:

$$
\begin{aligned}
\frac{\log |\mathcal{T}(\boldsymbol{c})|}{N} \geq \omega \cdot \mathrm{H}(\theta) & \\
& +\theta \omega\left(\mathrm{H}\left(\frac{1}{q-1}\right)+\frac{q-2}{q-1} \log (q-2)\right) \\
& +(1-\omega) \cdot \mathrm{H}(\theta)+\theta(1-\omega) \log (q-1) \\
& \quad-\frac{3 \log (N+1)}{N} \\
=\mathrm{H}(\theta) & +\theta \log (q-1)-\frac{3 \log (N+1)}{N} .
\end{aligned}
$$

On the other hand, the set, $\mathcal{Y}$, of all words $y \in F^{N}$ of weight $\theta N$ has size

$$
|\mathcal{Y}|=\left(\begin{array}{c}
N \\
\theta N
\end{array}\right)(q-1)^{\theta N}
$$

and, so,

$$
\frac{\log |\mathcal{Y}|}{N} \leq \mathrm{H}(\theta)+\theta \log (q-1)
$$


Combining (46) and 477 we conclude that

$$
\frac{\mathcal{T}(\boldsymbol{c})}{|\mathcal{Y}|} \geq \frac{1}{(N+1)^{3}} \text {. }
$$

For $\boldsymbol{y} \in \mathcal{Y}$, let

$$
\mathcal{C}(\boldsymbol{y})=\{\boldsymbol{c} \in \mathcal{C}: \boldsymbol{y} \in \mathcal{T}(\boldsymbol{c})\} .
$$

The set $\mathcal{C}^{*}(\boldsymbol{y})=\boldsymbol{y}+\mathcal{C}(\boldsymbol{y})$ forms a constant-weight code of codeword weight $\omega^{*} N$ (and of the same minimum distance $d$ as $\mathcal{C}$ ). Summing now on the size of $\mathcal{C}^{*}(\boldsymbol{y})$ over all $\boldsymbol{y} \in \mathcal{Y}$ yields:

$$
\begin{aligned}
\sum_{\boldsymbol{y} \in \mathcal{Y}}\left|\mathcal{C}^{*}(\boldsymbol{y})\right| & =\sum_{\boldsymbol{y} \in \mathcal{Y}}|\mathcal{C}(\boldsymbol{y})| \\
& =|\{(\boldsymbol{y}, \boldsymbol{c}) \in \mathcal{Y} \times \mathcal{C}: \boldsymbol{y} \in \mathcal{T}(\boldsymbol{c})\}| \\
& =\sum_{\boldsymbol{c} \in \mathcal{C}}|\mathcal{T}(\boldsymbol{c})|
\end{aligned}
$$

and, so,

$$
\frac{1}{|\mathcal{Y}|} \sum_{\boldsymbol{y} \in \mathcal{Y}}\left|\mathcal{C}^{*}(\boldsymbol{y})\right|=\frac{1}{|\mathcal{Y}|} \sum_{\boldsymbol{c} \in \mathcal{C}}|\mathcal{T}(\boldsymbol{c})| \stackrel{48}{\geq} \frac{|\mathcal{C}|}{(N+1)^{3}} .
$$

Hence, there must be at least one word $y \in \mathcal{Y}$ for which $\mathcal{C}^{*}=\mathcal{C}^{*}(\boldsymbol{y})$ satisfies (44).

\section{APPENDIX B \\ Characterization of $\bar{R}_{0}(\omega, n, \mu)$}

We show here how to compute the expression (15). It is easy to see that this expression equals $(n-1) / n$ when $\omega=0$ (since $\boldsymbol{\vartheta}$ is forced then to be all-zero on the support of $\pi$ ) and it vanishes when $\omega \geq(q-1) / q$ (by taking $\vartheta_{s}=\omega$ for all $s \in[n])$. Hence, we can assume from now on that $\omega \in$ $(0,(q-1) / q)$.

We introduce the following notation. For $n \in \mathbb{Z}^{+}$and $\omega \in$ $[0,(q-1) / q]$, let $\zeta_{n}(\omega)$ denote a particular minimizing $z$ of the expression for $\lambda(\omega, n)$ in (5). Also, for $n \in \mathbb{Z}^{+}$, let the polynomials $P_{n}(z)$ and $Q_{n}(z)$ be defined by

$$
\begin{aligned}
& P_{n}(z)=-(q-1) \cdot\left((1+(q-1) z)^{n-1}-(1-z)^{n-1}\right) \\
& Q_{n}(z)=(1+(q-1) z)^{n}+(q-1)(1-z)^{n}
\end{aligned}
$$

(note that $n \cdot P_{n}(z)$ is the derivative of $Q_{n}(z)$ ). Then (5) can be written as

$$
\begin{aligned}
\lambda(\omega, n) & =\inf _{z \in(0,1]}\left\{-\omega \log _{q} z-\frac{1}{n}+\frac{1}{n} \log _{q} Q_{n}(z)\right\} \\
& =-\omega \log _{q} \zeta_{n}(\omega)-\frac{1}{n}+\frac{1}{n} \log _{q} Q_{n}\left(\zeta_{n}(\omega)\right) .
\end{aligned}
$$

We have the following lemma.

Lemma 18. For $n>1$ and $\omega \in[0,(q-1) / q]$, the value $\zeta_{n}(\omega)$ is the unique real root in $[0,1]$ of the polynomial

$$
U_{\omega, n}(z)=\omega \cdot Q_{n}(z)-z \cdot P_{n}(z) .
$$

Moreover, the mapping $\omega \mapsto \zeta_{n}(\omega)$ is strictly increasing.

Proof. It follows from the proof of Lemma 4 that $\zeta_{n}(0)=$ 0 and $\zeta_{n}((q-1) / q)=1$. Assuming hereafter that $\omega \in$ $(0,(q-1) / q)$, it also follows from that proof that $\zeta_{n}(\omega)$ is an (interior) point in $(0,1)$; as such, it is a local minimum of the objective function in (49) and, so, it equals a value $z$ at which the derivative of that function (with respect to $z$ ) vanishes:

$$
\frac{\omega}{z}-\frac{P_{n}(z)}{Q_{n}(z)}=0
$$

This equation, which is equivalent to requiring that $U_{\omega, n}(z)=$ 0 , can be rearranged into

$$
\frac{(1-\omega)(q-1) z-\omega}{(1-\omega) z+\omega}=(q-1) \cdot\left(\frac{1-z}{1+(q-1) z}\right)^{n-1} .
$$

In the range $z \in[0,1]$, the left-hand side of 51 is strictly increasing in $z$ (from the value -1 at $z=0$ to $q-1-q \omega$ at $z=1$ ) while the right-hand side of (51) is strictly decreasing in $z$. Hence, for any $\omega \in(0,(q-1) / q)$, there is (at most) one $z \in[0,1]$ that satisfies (51), and $\zeta_{n}(\omega)$ must then be that $z$. Moreover, since the left-hand side of (51) is a strictly decreasing expression in $\omega$, the mapping $\omega \mapsto \zeta_{n}(\omega)$ is strictly increasing.

Remark 8. By [50), the inverse mapping $z \mapsto \omega=\zeta_{n}^{-1}(z)$ is given by

$$
\zeta_{n}^{-1}(z)=\frac{z \cdot P_{n}(z)}{Q_{n}(z)} .
$$

Note also that Lemma 18 is false when $n=1$. In this case $\zeta_{1}(0)$ is arbitrary (and $U_{0,1}(z)$ is identically zero) while $\zeta_{1}(\omega)=1$ when $\omega>0$ (it is then a global-rather than local-minimum of the objective function in (49)).

We proceed to the characterization of the minimum in the inner expression in (15). We will use the notation $[n]^{*}$ for the set $[n] \backslash\{1\}$.

Lemma 19. Given $n \in \mathbb{Z}^{+}, \mu \in[n]$, and $\omega \in(0,(q-1) / q)$, let $\boldsymbol{\pi}=\left(\pi_{s}\right)_{s \in[n]} \in \mathbb{R}_{\geq 0}^{n}$ be a vector that satisfies conditions $(P 1)-(P 2)$ with support $\mathcal{S}=\operatorname{Supp}(\boldsymbol{\pi})$. A minimizer of the inner expression in (15) under the constraint $(P 3)$ is any vector $\vartheta=\left(\vartheta_{s}\right)_{s \in[n]} \in \mathbb{R}_{\geq 0}^{n}$ whose subvector $\left(\vartheta_{s}\right)_{s \in \mathcal{S}}$ is uniquely determined as follows: $\vartheta_{s}=\zeta_{s}^{-1}\left(z^{*}\right)$, where $z^{*}$ is the unique real in $[0,1]$ that satisfies

$$
\sum_{s \in \mathcal{S}} \pi_{s} \cdot \zeta_{s}^{-1}\left(z^{*}\right)=\omega
$$

(taking $\zeta_{1}^{-1}\left(z^{*}\right) \equiv 0$ unless $\mathcal{S}=\{1\}$, in which case $\vartheta_{1}=\omega$ ).

Proof. Since $\omega \mapsto R_{0}(\omega, 1)$ is identically zero, the lemma holds when $\mathcal{S}=\{1\}$, so we assume hereafter that $\mathcal{S} \neq\{1\}$ and denote $\mathcal{S}^{*}=\mathcal{S} \backslash\{1\}$. By condition (P3), we can further assume that $\vartheta_{1}=0$, since otherwise we can reduce $\vartheta_{1}$ and increase $\vartheta_{s}$ for $s \in[n]^{*}$, thereby only decreasing the inner expression in 15).

Define the function $\boldsymbol{\vartheta}=\left(\vartheta_{s}\right)_{s \in[n]^{*}} \mapsto f(\boldsymbol{\vartheta})$ for every $\boldsymbol{\vartheta} \in$ $\mathbb{R}_{\geq 0}^{n-1}$ to be the minimand in 15 :

$$
f(\boldsymbol{\vartheta})=f_{\boldsymbol{\pi}}(\boldsymbol{\vartheta})=\sum_{s \in \mathcal{S}^{*}} \pi_{s} \cdot \boldsymbol{R}_{0}\left(\vartheta_{s}, s\right) .
$$

Since $\vartheta \mapsto R_{0}(\vartheta, s)$ is convex, so is $\vartheta \mapsto f(\vartheta)$. 
We minimize $f$ subject to condition (P3) using the method of Lagrange multipliers [17, §10.3]: we introduce a variable $\xi$ and require that the partial derivatives of the Lagrangian

$$
L(\boldsymbol{\vartheta}, \xi)=f(\boldsymbol{\vartheta})+\xi \cdot\left(\omega-\sum_{s \in[n]^{*}} \pi_{s} \cdot \vartheta_{s}\right)
$$

be zero with respect to $\xi$ and the entries of $\left(\vartheta_{s}\right)_{s \in \mathcal{S}^{*}}$, namely,

$$
\sum_{s \in \mathcal{S}^{*}} \pi_{s} \cdot \vartheta_{s}=\omega
$$

and

$$
\frac{\partial}{\partial \vartheta_{s}} L(\vartheta, \xi)=0, \quad s \in \mathcal{S}^{*}
$$

Denoting $\boldsymbol{\zeta}(\boldsymbol{\vartheta})=\left(\zeta_{s}\left(\vartheta_{s}\right)\right)_{s \in \mathcal{S}^{*}}$, we can write $f(\boldsymbol{\vartheta})$ as:

$$
f(\boldsymbol{\vartheta})=\left.f(\boldsymbol{\vartheta}, \boldsymbol{z})\right|_{\boldsymbol{z}=\boldsymbol{\zeta ( \boldsymbol { \vartheta } )}},
$$

where $\boldsymbol{z}=\left(z_{s}\right)_{s \in \mathcal{S}^{*}}$ is a real vector of variables and

$$
f(\boldsymbol{\vartheta}, \boldsymbol{z})=1+\sum_{s \in \mathcal{S}^{*}} \pi_{s}\left(\vartheta_{s} \cdot \log _{q} z_{s}-\frac{1}{s} \log _{q} Q_{s}\left(z_{s}\right)\right) .
$$

Recalling that

$$
\left.\frac{\partial}{\partial z_{i}} f(\boldsymbol{\vartheta}, \boldsymbol{z})\right|_{\boldsymbol{z}=\boldsymbol{\zeta}(\boldsymbol{\vartheta})}=0, \quad \text { for every } i \in \mathcal{S}^{*}
$$

we get for every $s \in \mathcal{S}^{*}$ :

$$
\begin{aligned}
\frac{\partial}{\partial \vartheta_{s}} f(\boldsymbol{\vartheta})= & \left.\frac{\partial}{\partial \vartheta_{s}} f(\boldsymbol{\vartheta}, \boldsymbol{z})\right|_{\boldsymbol{z}=\boldsymbol{\zeta}(\boldsymbol{\vartheta})} \\
& +\sum_{i \in \mathcal{S}^{*}} \underbrace{\left.\frac{\partial}{\partial z_{i}} f(\boldsymbol{\vartheta}, \boldsymbol{z})\right|_{\boldsymbol{z}=\boldsymbol{\zeta}(\boldsymbol{\vartheta})}}_{0} \cdot \frac{\partial}{\partial \vartheta_{s}} \zeta_{i}\left(\vartheta_{i}\right) \\
= & \pi_{s} \cdot \log _{q} \zeta_{s}\left(\vartheta_{s}\right) .
\end{aligned}
$$

Hence, by 53,

$$
\pi_{s} \cdot\left(\log _{q} \zeta_{s}\left(\vartheta_{s}\right)-\xi\right)=0,
$$

namely, the values $\zeta_{s}\left(\vartheta_{s}\right)$ are equal to (the same value) $z^{*}=$ $q^{\xi}$, for all $s \in \mathcal{S}^{*}$. Finally, by (52), the value $z^{*}$ must be such that

$$
\sum_{s \in \mathcal{S}^{*}} \pi_{s} \cdot \zeta_{s}^{-1}\left(z^{*}\right)=\omega
$$

This equality determines $z^{*}$ uniquely, since $z \mapsto \zeta_{s}^{-1}(z)$ is strictly increasing for any $s \in[n]^{*}$.

We next turn to the characterization of the outer maximum in 15 .

Lemma 20. Given $n \in \mathbb{Z}^{+}, \mu \in[n]$, and $\omega \in(0,(q-1) / q)$, let $k=\lfloor\mu\rfloor$ and

$$
\pi=\frac{k(k+1)}{\mu}-k .
$$

The entries of the maximizing vector $\boldsymbol{\pi}=\left(\pi_{s}\right)_{s \in[n]} \in \mathbb{R}_{\geq 0}^{n}$ in (15) under the constraints $(P 1)-(P 2)$ are all zero, except for the entries that are indexed by $k$ and (possibly) $k+1$, where

$$
\pi_{k}=\pi \quad \text { and } \quad \pi_{k+1}=1-\pi .
$$

Proof. When $\mu=1$, conditions (P1)-(P2) force $\pi$ to be $(100 \ldots 0)$ (i.e., $\operatorname{Supp}(\boldsymbol{\pi})=\{1\})$, in which case $\omega \mapsto$ $\bar{R}_{0}(\omega, n, 1)$ is identically zero. Hence, we assume hereafter that $\mu>1$, in which case a maximizing $\pi$ must have support $\neq\{1\}$ to achieve $\bar{R}_{0}(\omega, n, \mu)>0$.

Define the function $\boldsymbol{\pi}=\left(\pi_{s}\right)_{s \in[n]} \mapsto g(\boldsymbol{\pi})$ for every $\boldsymbol{\pi} \in$ $\mathbb{R}_{\geq 0}^{n}$ to be the maximand in 15 , namely,

$$
\begin{aligned}
g(\boldsymbol{\pi}) & =\sum_{s \in[n]} \pi_{s} \cdot R_{0}\left(\vartheta_{s}^{*}, s\right) \\
& =1-\omega \cdot \log _{q} z^{*}+\sum_{s \in[n]} \frac{\pi_{s}}{s} \log _{q} Q_{s}\left(z^{*}\right),
\end{aligned}
$$

where $\vartheta_{s}^{*}=\zeta_{s}^{-1}\left(z^{*}\right)$ for each $s \in[n]$ and $z^{*}$ is as in Lemma 19, namely, $z^{*}$ is determined uniquely by $\pi$ (and $\omega$ ) and, therefore, so is each $\vartheta_{s}^{*}$ (in particular, $\vartheta_{1}^{*}=0$ ). We do the maximization subject to the constraints (P1)-(P2) using the Kuhn-Tucker conditions [17, §10.8]: we introduce a real variable $\xi$ and require that the partial derivatives of

$$
\begin{aligned}
K(\boldsymbol{\pi}, \xi)= & g(\boldsymbol{\pi})+\xi \cdot\left(1-\sum_{s \in[n]} \pi_{s}\right) \\
& -\beta \cdot\left(\frac{1}{\mu}-\sum_{s \in[n]} \frac{\pi_{s}}{s}\right)+\sum_{s \in[n]} \eta_{s} \cdot \pi_{s}
\end{aligned}
$$

be zero with respect to $\xi$ and the entries of $\pi$, for some nonnegative $\beta$ and $\boldsymbol{\eta}=\left(\eta_{s}\right)_{s \in[n]}$ that satisfy

$$
\beta \cdot\left(\frac{1}{\mu}-\sum_{s \in[n]} \frac{\pi_{s}}{s}\right)=0
$$

and

$$
\eta_{s} \cdot \pi_{s}=0, \quad \text { for each } s \in[n] .
$$

The second term in the right-hand side of (55) corresponds to condition (P1); the third term and (56) correspond to condition (P2); and the last term and (57) correspond to requiring that $\pi$ be nonnegative.

Similarly to what we have done in the proof of Lemma 19 we can write $g(\boldsymbol{\pi})$ as

$$
g(\boldsymbol{\pi})=\left.g(\boldsymbol{\pi}, \boldsymbol{z})\right|_{\boldsymbol{z}=z^{*} \cdot \mathbf{1}},
$$

where $z=\left(z_{s}\right)_{s \in[n]^{*}}$ is a real vector of variables and $\mathbf{1}$ stands for the all-one vector in $\mathbb{R}_{\geq 0}^{n-1}$. Recalling that

$$
\left.\frac{\partial}{\partial z_{i}} g(\boldsymbol{\pi}, \boldsymbol{z})\right|_{z_{i}=z^{*}}=0, \quad \text { for every } i \in[n]^{*},
$$

we get:

$$
\begin{aligned}
\frac{\partial}{\partial \pi_{s}} g(\boldsymbol{\pi})= & \left.\frac{\partial}{\partial \pi_{s}} g(\boldsymbol{\pi}, \boldsymbol{z})\right|_{\boldsymbol{z}=z^{*} \cdot \mathbf{1}} \\
& \quad+\sum_{i \in[n] *} \underbrace{\left.\frac{\partial}{\partial z_{i}} g(\boldsymbol{\pi}, \boldsymbol{z})\right|_{z_{i}=z^{*}}}_{0} \cdot \frac{\partial}{\partial \pi_{s}} \zeta_{i}\left(\vartheta_{i}^{*}(\boldsymbol{\pi})\right) \\
= & \frac{1}{s} \log _{q} Q_{s}\left(z^{*}\right) .
\end{aligned}
$$

Returning to the expression for $K(\boldsymbol{\pi}, \xi)$ in (55), we conclude that $(\partial K(\boldsymbol{\pi}, \xi)) / \partial \pi_{s}=0$ translates into

$$
\eta_{s}=\psi(s)
$$

where

$$
\psi(s)=\psi(s, \beta)=\frac{1}{s}\left(\log _{q} Q_{s}\left(z^{*}\right)-\beta\right)+\xi .
$$


Next, we analyze the function $s \mapsto \psi(s)$ in the real variable $s \in[1, \infty)$. Taking the derivative of this function, we get:

$$
\begin{aligned}
\psi^{\prime}(s)=\frac{1}{s} & \cdot \frac{1}{Q_{s}\left(z^{*}\right)}\left(\left(1+(q-1) z^{*}\right)^{s} \log _{q}\left(1+(q-1) z^{*}\right)\right. \\
& \left.\quad+(q-1)\left(1-z^{*}\right)^{s} \log _{q}\left(1-z^{*}\right)\right) \\
& \quad-\frac{1}{s^{2}}\left(\log _{q} Q_{s}\left(z^{*}\right)-\beta\right) \\
= & \frac{1}{s^{2}}\left(\beta-\mathrm{H}_{q}\left(p_{s}\right)\right)
\end{aligned}
$$

where

$$
p_{s}=\frac{(q-1)\left(1-z^{*}\right)^{s}}{Q_{s}\left(z^{*}\right)}
$$

and $x \mapsto \mathrm{H}_{q}(x)$ is the $q$-ary entropy function $x \cdot \log _{q}(q-1)-$ $x \log _{q} x-(1-x) \log _{q}(1-x)$. This function is strictly increasing on $[0,(q-1) / q]$ and $s \mapsto p_{s}$ is strictly decreasing on $[1, \infty)$, with the following maximum value attained at $s=1$ :

$$
p_{1}=\frac{(q-1)\left(1-z^{*}\right)}{Q_{1}\left(z^{*}\right)}<\frac{q-1}{q} .
$$

We conclude from (59) that, on the interval $[1, n]$, the function $s \mapsto \psi(s)$ is either (i) strictly increasing, or (ii) strictly decreasing, or (iii) unimodal with one local minimum. Note from (59) that $\beta=0$ implies case (ii).

As our next step, we turn back to (58). Since $\eta_{s} \geq 0$ for all $s \in[n]$, in case (i) we can have $\eta_{s}=0$ only when $s=1$. Similarly, in case (ii) we can have $\eta_{s}=0$ only when $s=n$. And in case (iii) we can have $\eta_{s}=0$ only for the (one or) two consecutive indexes $s$ that are adjacent to the minimum of $\psi(\cdot)$. We conclude that $\eta_{s}>0$ for all $s$ except for those two indexes; this, together with (57), implies that the support of a maximizing $\pi$ contains (only) those two indexes.

Observe that by (56), a maximizing $\pi$ must satisfy condition (P2) with equality, unless $\beta=0$, in which case $\boldsymbol{\pi}=\left(\begin{array}{llll}0 & 0 & 0 & \ldots\end{array}\right)$ (case (ii) above). Condition (P2) would then read $1 / n=\pi_{n} / n \geq 1 / \mu$, i.e., $\mu \geq n$. Yet we assume that $\mu \in[n]$, thereby forcing $\mu=n$, so condition (P2) holds with equality in this case too.

Finally, let $k$ and (possibly) $k+1$ be the elements in the support of a maximizing $\pi$. We then get from conditions (P1) (and the equality version of) (P2) that the entries $\pi_{k}$ and $\pi_{k+1}$ satisfy the following equations:

$$
\begin{aligned}
\pi_{k}+\pi_{k+1} & =1 \\
\frac{\pi_{k}}{k}+\frac{\pi_{k+1}}{k+1} & =\frac{1}{\mu} .
\end{aligned}
$$

Solving these equations yields $\pi_{k}=\pi$ and $\pi_{k+1}=1-\pi$, where $\pi$ is given by (54), and the value of $k$ is determined by

$$
\begin{aligned}
& \pi \geq 0 \quad \Rightarrow \quad \mu \leq k+1 \\
& \pi \leq 1 \quad \Rightarrow \quad \mu \geq k .
\end{aligned}
$$

Combining Lemmas 19 and 20 leads to the following recipe for computing the value of $\bar{R}_{0}(\omega, n, \mu)$.
Proposition 21. Given $n \in \mathbb{Z}^{+}, \mu \in[n]$, and $\omega \in$ $[0,(q-1) / q]$, let $k=\lfloor\mu\rfloor$ and let $\pi$ be as in (54). Also, let $z^{*}$ be the unique real in $[0,1]$ that satisfies

$$
\pi \cdot \zeta_{k}^{-1}\left(z^{*}\right)+(1-\pi) \cdot \zeta_{k+1}^{-1}\left(z^{*}\right)=\omega
$$

(taking $\zeta_{1}^{-1}\left(z^{*}\right) \equiv 0$ when $\mu \in(1,2)$ and $\zeta_{1}^{-1}\left(z^{*}\right)=\omega$ when $\mu=1)$. Then

$$
\bar{R}_{0}(\omega, n, \mu)=\pi \cdot R_{0}(\vartheta, k)+(1-\pi) \cdot R_{0}\left(\vartheta^{\prime}, k+1\right),
$$

where

$$
\vartheta=\zeta_{k}^{-1}\left(z^{*}\right) \quad \text { and } \quad \vartheta^{\prime}=\zeta_{k+1}^{-1}\left(z^{*}\right) .
$$

In particular, when $\mu$ is an integer then

$$
\bar{R}_{0}(\omega, n, \mu)=R_{0}(\omega, \mu) .
$$

Remark 9. By Remark 8, solving (60) for $z^{*}$ amounts to finding the (unique) real root in $[0,1]$ of the polynomial

$$
\begin{aligned}
& \omega \cdot Q_{k}(z) \cdot Q_{k+1}(z) \\
& \quad-z \cdot\left(\pi \cdot P_{k}(z) \cdot Q_{k+1}(z)+(1-\pi) \cdot P_{k+1}(z) \cdot Q_{k}(z)\right) .
\end{aligned}
$$

Remark 10. Using Proposition 21, it is fairly easy to compute the maximization in (41) numerically. Given $n \in \mathbb{Z}^{+}$, we next argue that for sufficiently small positive $\omega$ (within an interval whose length depends on $n$ ), the maximum in (41) is attained at $\nu=0$. For any $\nu \in[0,(n-1) /(n+1)]$, let $\mu(\nu)$ denote the value $((1-\nu) /(1+\nu)) n(\in[n])$. Given $\omega \in[0,2(q-1) /(q(n+1))]$, the objective function in (41) can be verified to be (continuous and) piecewise differentiable in $\nu$. Specifically, at any $\nu \in[0,(n-1) /(n+1)]$ for which $\mu(\nu)$ is not an integer, the derivative is given by

$$
\left(1+\frac{k}{n}\right) \log _{q} Q_{k+1}\left(z^{*}\right)-\left(1+\frac{k+1}{n}\right) \log _{q} Q_{k}\left(z^{*}\right),
$$

where $k$ and $z^{*}$ are as in Proposition 21, with $\mu$ and $\omega$ therein taken as $\mu=\mu(\nu)$ and $\omega /(1-\nu)(\in[0,(q-1) / q])$, respectively. Now, by (50) and (60) it follows that $z^{*} \rightarrow 0$ as $\omega \rightarrow 0$ (uniformly in $\nu$ ). And since the expression 61) is negative at $z^{*}=0$ for any $k \in[n-1]$, we conclude that for sufficiently small $\omega>0$, the objective function in (41) is decreasing in $\nu$. Thus, the maximum therein is attained at $\nu=0$, in which case

$$
\widehat{R}_{0}(\omega, n)=R_{0}(\omega, n) .
$$

Remark 11. As we pointed out in Remark 5, the value $\bar{R}_{0}(\delta / 2, n, \mu)$ bounds from above the rate of any all-disjoint linearly recoverable $(\delta, n)$-LRC sequence $\left(\mathrm{C}_{i}\right)_{i=1}^{\infty}$ under the additional condition that the average size of the repair groups of each $C_{i}$ is at most $\mu$ (in the limit when $i \rightarrow \infty$ ). A related relevant problem is obtaining such a bound when the average is computed per coordinate, i.e., the average is taken over the whole list of the $N_{i}$ repair groups of $\mathrm{C}_{i}$; thus, each of the distinct repair groups is counted a number of times which equals the number of coordinates that it covers (namely, its size). A counterpart of the bound (2) for this setting was presented in [24]. 
A sphere-packing upper bound for this setting is obtained by substituting $\omega=\delta / 2$ in (15), except that condition (P2) is replaced by

$$
\text { P2) } \sum_{s \in[n]} s \cdot \pi_{s} \leq \mu \text {. }
$$

Proposition 21 still holds, except that the value of $\pi$ in 54 is changed into

$$
\pi=k+1-\mu \text {. }
$$

Specifically, Lemma 19 holds as is; as for Lemma 20, the third term in (55) (and, accordingly, the left-hand side of (56)) is replaced by

$$
\beta \cdot\left(\mu-\sum_{s \in[n]} s \cdot \pi_{s}\right)
$$

(with a plus sign). Consequently, 59 becomes

$$
\beta-\frac{1}{s^{2}} \cdot \mathrm{H}_{q}\left(p_{s}\right)
$$

which, in turn, leads to the same conclusions about the function $s \mapsto \psi(s)$ (cases (i)-(iii)). Thus, the support of a maximizing $\pi$ contains up to two indexes, $k$ and $k+1$, and, by (P1)-(P2), the values of $\pi_{k}$ and $\pi_{k+1}$ are the solutions of

$$
\begin{gathered}
\pi_{k}+\pi_{k+1}=1 \\
k \cdot \pi_{k}+(k+1) \cdot \pi_{k+1}=\mu .
\end{gathered}
$$

\section{REFERENCES}

[1] M. Aaltonen, "A new upper bound on nonbinary block codes," Discrete Math., 83 (1990) 139-160.

[2] A. Agarwal, A. Barg, S. Hu, A. Mazumdar, I. Tamo, "Combinatorial alphabet-dependent bounds for locally recoverable codes," IEEE Trans. Inf. Theory, 64 (2018), 3481-3492.

[3] S.B. Balaji, P.V. Kumar, "Bounds on the rate and minimum distance of codes with availability," Proc. 2017 IEEE Int'l Symp. Inf. Theory (ISIT 2017), Aachen, Germany (June 2017), 3155-3159.

[4] Y. Ben-Haim, S. Litsyn, "A new upper bound on the rate of non-binary codes," Adv. Math. Commun., 1 (2007), 83-92.

[5] Y. Ben-Haim, S. Litsyn, private communication, 2020.

[6] C. Bachoc, V. Chandar, G. Cohen, P. Solé, A. Tchamkerten, "On balanced weight codes," IEEE Trans. Inf. Theory, 57 (2011), 6780-6787.

[7] M. Blaum, "Extended integrated interleaved codes over any field with applications to locally recoverable codes," IEEE Trans. Inf. Theory, 66 (2020), 936-956.

[8] V. Cadambe, A. Mazumdar, "Bounds on the size of locally recoverable codes," IEEE Trans. Inf. Theory, 61 (2015), 5787-5794.

[9] A. Dembo, O. Zeitouni, Large Deviations Techniques and Applications, Second Edition, Springer, Berlin, 2010.

[10] P. Gopalan, C. Huang, H. Simitci, S. Yekhanin, "On the locality of codeword symbols," IEEE Trans. Inf. Theory, 58 (2012), 6925-6934.

[11] S. Gopi, V. Guruswami, S. Yekhanin, "Maximally recoverable LRCs: A field size lower bound and constructions for few heavy parities," IEEE Trans. Inf. Theory, 66 (2020), 6066-6083.

[12] M. Grezet, R. Freij-Hollanti, T. Westerbäck, C. Hollanti, "Alphabetdependent bounds for linear locally repairable codes based on residual codes," IEEE Trans. Inf. Theory, 65 (2019), 6089-6100.

[13] V. Guruswami, L. Jin, C. Xing, "Constructions of maximally recoverable local reconstruction codes via function fields," IEEE Trans. Inf. Theory, to appear.

[14] P. Huang, E. Yaakobi, P.H. Siegel, "Multi-erasure locally recoverable codes over small fields: A tensor product approach," IEEE Trans. Inf. Theory, 66 (2020), 2609-2624.

[15] P. Huang, E. Yaakobi, H. Uchikawa, P.H. Siegel, "Binary linear locally repairable codes," IEEE Trans. Inf. Theory, 62 (2016), 6268-6283.

[16] T. Laihonen, S. Litsyn, "On upper bounds for minimum distance and covering radius of non-binary codes," Des. Codes, Cryptogr., 14 (1998), 71--80.
[17] D.G. Luenberger Linear and Nonlinear Programming, Second Edition, Addison-Wesley, Reading, Massachusetts, 1984.

[18] R.J. McEliece, E.R. Rodemich, H. Rumsey, Jr., L.R. Welch, "New upper bounds on the rate of a code via the Delsarte-MacWilliams inequalities," IEEE Trans. Inf. Theory, 23 (1977), 157-166.

[19] J. Ma, G. Ge, "Optimal binary linear locally repairable codes with disjoint repair groups," SIAM J. Discrete Math., 33 (2019), 2509-2529.

[20] L. Pamies-Juarez, H.D.L. Hollmann, F. Oggier, "Locally repairable codes with multiple repair alternatives," Proc. 2013 IEEE Int'l Symp. Inf. Theory (ISIT 2013), Istanbul, Turkey (July 2013), 892-896.

[21] D.S. Papailiopoulos, A.G. Dimakis, "Locally repairable codes," IEEE Trans. Inf. Theory, 60 (2014), 5843-5855.

[22] N. Prakash, G.M. Kamath, V. Lalitha, P.V. Kumar, "Optimal linear codes with a local-error-correction property," Proc. 2012 IEEE Int'l Symp. Inf. Theory (ISIT 2012), Cambridge, Massachusetts (July 2012), 2776-2780.

[23] R.M. Roth, Introduction to Coding Theory, Cambridge University Press, Cambridge, UK, 2006.

[24] M. Shahabinejad, M. Khabbazian, M. Ardakani, "On the average locality of locally repairable codes," IEEE Trans. Commun., 66 (2018), 27732783.

[25] I. Tamo, A. Barg, A. Frolov, "Bounds on the parameters of locally recoverable codes," IEEE Trans. Inf. Theory, 62 (2016), 3070-3083.

[26] I. Tamo, A. Barg, "A family of optimal locally recoverable codes," IEEE Trans. Inf. Theory, 60 (2014), 4661-4676.

[27] A. Wang, Z. Zhang, "Repair locality with multiple erasure tolerance," IEEE Trans. Inf. Theory, 60 (2014), 6979-6987.

[28] A. Wang, Z. Zhang, D. Lin, "Bounds for binary linear locally repairable codes via a sphere-packing approach," IEEE Trans. Inf. Theory, 65 (2019), 4167-4179. 


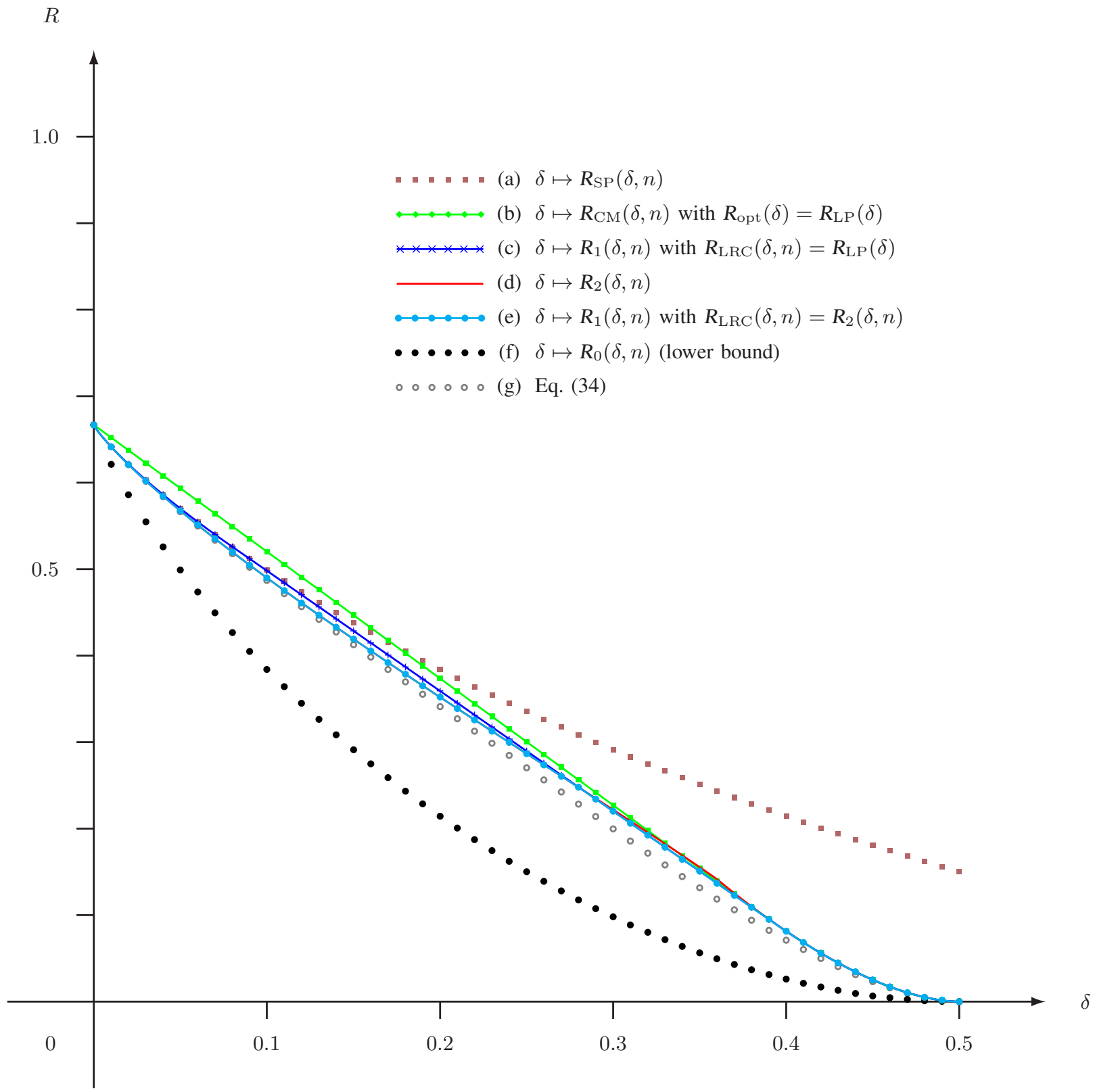

Fig. 2. Bounds for $q=2$ and $n=3$. 


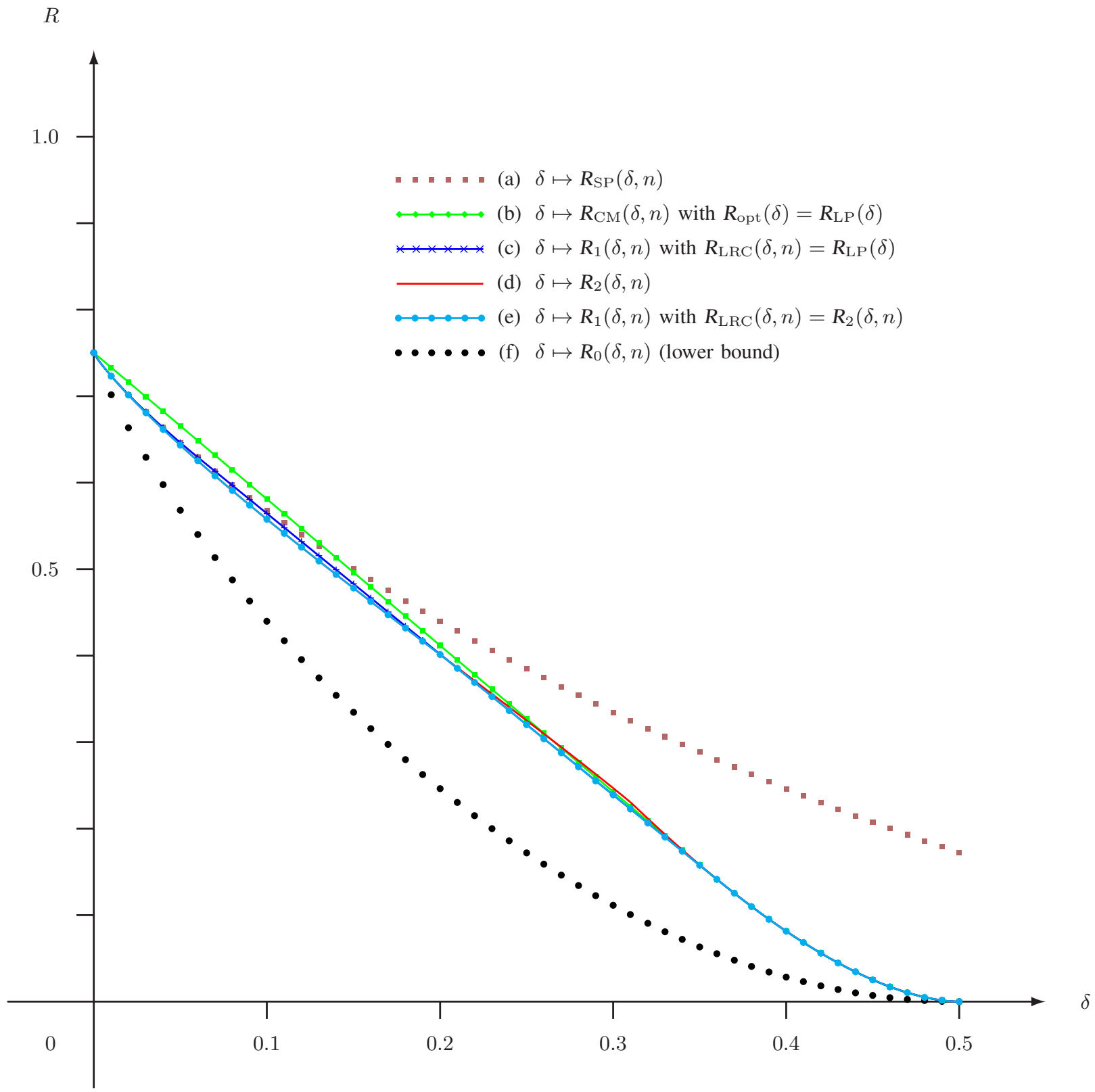

Fig. 3. Bounds for $q=2$ and $n=4$. 


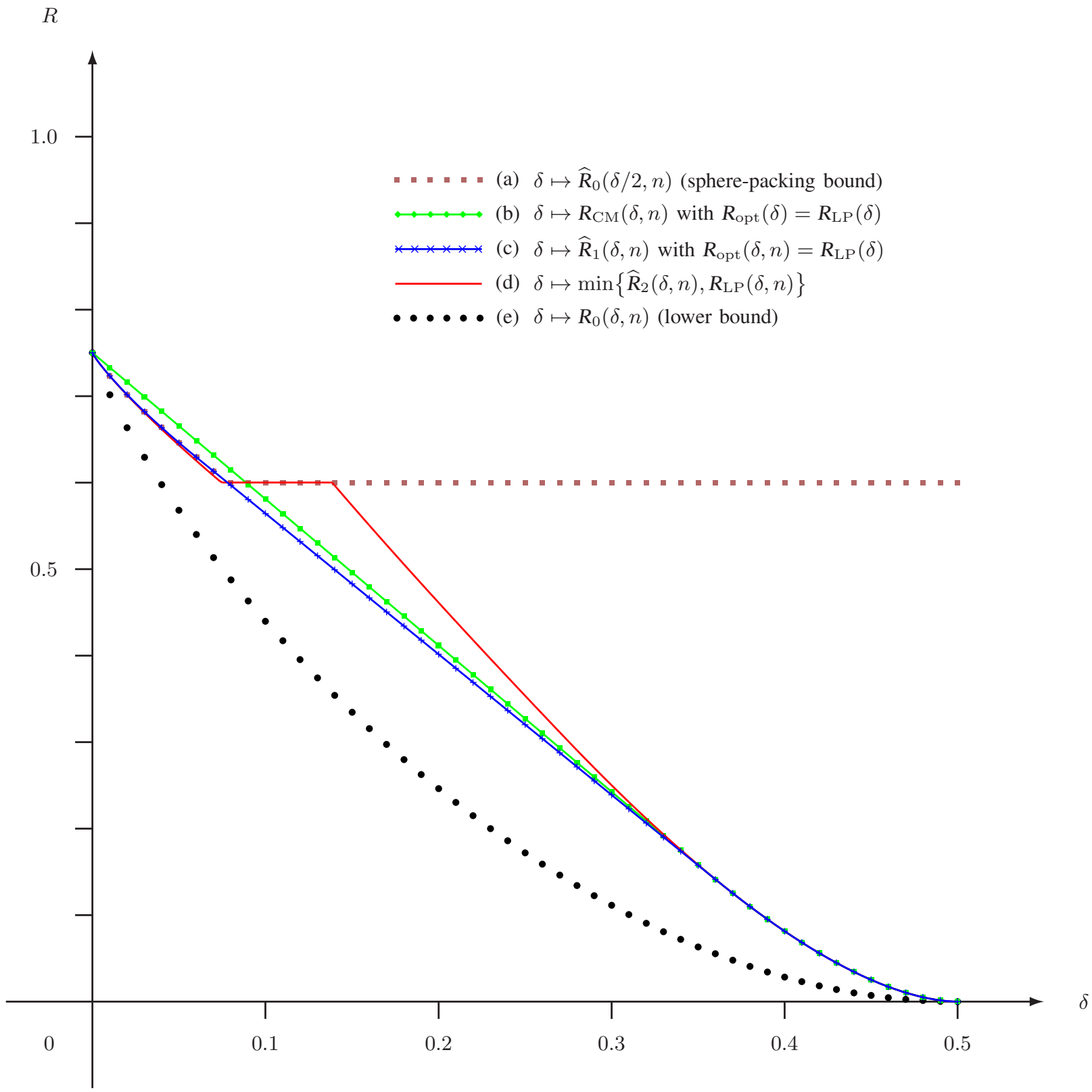

Fig. 4. Bounds for $q=2$ and $n=4$ (not necessarily all-disjoint). 